\title{
Mean Value Property and Harmonicity on Carnot-Carathéodory Groups
}

\author{
Tomasz Adamowicz ${ }^{1} \cdot$ Ben Warhurst ${ }^{2}$
}

Received: 3 March 2017 / Accepted: 10 October 2018 / Published online: 25 October 2018

(C) The Author(s) 2018

\begin{abstract}
We study strongly harmonic functions in Carnot-Carathéodory groups defined via the mean value property with respect to the Lebesgue measure. For such functions we show their Sobolev regularity and smoothness. Moreover, we prove that strongly harmonic functions satisfy the sub-Laplace equation for the appropriate gauge norm and that the inclusion is sharp. We observe that appropriate spherical harmonic polynomials in $\mathbb{H}_{1}$ are both strongly harmonic and satisfy the sub-Laplace equation. Our presentation is illustrated by examples.
\end{abstract}

Keywords Carnot group · Harmonic $\cdot$ Heisenberg group $\cdot$ Lie algebra $\cdot$ Lie group Laplace $\cdot$ Maximum principle $\cdot$ Mean value property $\cdot$ Strongly harmonic $\cdot$ Subelliptic equation $\cdot$ Sub-Riemannian $\cdot$ Weakly harmonic

Mathematics Subject Classification (2010) Primary: 35H20 - Secondary: 31E05, 53C17

\section{Introduction}

The main subject of our studies are harmonic functions on Carnot-Carathéodory groups with emphasis on the setting of Heisenberg groups since in this case the pseudodistance induced by the fundamental solution of the sub-Laplacian is in fact a metric (see below for relevant definitions). Following works $[1,11]$ we define harmonic functions via the mean value property with respect to the underlying measure, i.e. we call a locally integrable

T. Adamowicz and B. Warhurst were supported by a grant Iuventus Plus of the Ministry of Science and Higher Education of the Republic of Poland, Nr 0009/IP3/2015/73.

\footnotetext{
Tomasz Adamowicz

T.Adamowicz@impan.pl

Ben Warhurst

B.Warhurst@mimuw.edu.pl
}

1 Institute of Mathematics, Polish Academy of Sciences, ul. Śniadeckich 8, 00-656 Warsaw, Poland

2 Institute of Mathematics, University of Warsaw, ul.Banacha 2, 02-097 Warsaw, Poland 
function $f: \Omega \rightarrow \mathbb{R}$ strongly harmonic in $\Omega$, if the following inequality holds for all balls $B(p, r) \Subset \Omega$ with $p \in \Omega$ and $r>0$ :

$$
f(p)=f_{B(p, r)} f(q) d q
$$

Here, $\Omega$ stands for a domain in a given Carnot-Carathéodory group, $d q$ denotes the corresponding Lebesgue measure, and the balls $B(p, r)$ are defined with respect to a given metric on $\Omega$. We refer to Section 3.2 for further definitions and more on motivations for our investigations.

Harmonic functions considered in [1] in general metric measure spaces are only Hölder regular, e.g., on geodesic spaces equipped with doubling measures or measures satisfying the annular decay condition, see [1, Theorems 4.1, 4.2], or locally Lipschitz regular for uniform measures or doubling measures on spaces supporting a $(1, p)$-Poincaré inequality, see [1, Proposition 5.2, Theorem 5.1]. In the setting of Carnot groups, the group structure and the presence of the Euclidean coordinates allow us to expect that harmonic functions exhibit higher regularity properties. Indeed, in Section 4.1 we show that functions satisfying (1) belong to the horizontal Sobolev spaces $H W_{l o c}^{1, s}$ for any $s>1$, see Theorem 4.1. The proof relies on measure theoretic properties of harmonic functions. Furthermore, by using the convolution and scaling techniques available in Carnot groups, we show in Theorem 4.2 the smoothness of harmonic functions. It turns out that for the proof of smoothness, one needs (1) to hold only for balls defined by a pseudodistance, i.e. the triangle inequality for $d$ in Eq. 1 can be relaxed.

Another topic we are especially interested in, is the interplay between harmonic functions and solutions to the subelliptic Laplace equation on a Carnot group (called the $\mathcal{L}$-harmonic equation). In Theorem 4.3 we show that functions possessing property (1) satisfy the subLaplace equation provided that the balls in the mean value property are considered with respect to the pseudonorm given by the fundamental solution of the sub-Laplace operator. As a corollary of Theorem 4.3 we obtain a variant of the Hadamard three-spheres theorem for strongly harmonic functions. Let us also mention that a counterpart of Theorem 4.3 in more general metric spaces is not known and is a subject of an ongoing investigation to determine the relation between strongly harmonic functions and the $p$-harmonic functions defined as local minima of the $p$-Dirichlet energy with respect to weak upper gradients.

Another aspect of harmonicity studied in our work relates to the fact that the subelliptic harmonic functions are known to satisfy the kernel-type mean value property, see Formula (25) in Theorem 4.4 below and Appendix for its proof. Thus, we are also interested in studying the relation between this type of property and Eq. 1, see Section 4.4.

In Section 5 we show that the intersection of the class of $\mathcal{L}$-harmonic and strongly harmonic functions, considered with respect to the $\mathcal{L}$-gauge distance, contains a subclass of spherical harmonic polynomials, called for short, spherical harmonics. At the first glance it might be surprising that such a class exists, taking into account that a spherical harmonic must satisfy two types of mean value properties, namely the one given in Definition 3.2 and the one defined by Eq. 25. Moreover, we discuss an example of a spherical harmonic function (and thus an $\mathcal{L}$-harmonic function) which fails to be strongly harmonic, see Example 6 . Finally, in Section 5 we also propose two open questions on finding all spherical harmonics which are strongly harmonic.

In the last section of our work we briefly discuss a notion of determining set and prove that under additional assumptions, a dense subset of a domain in $\mathbb{H}_{1}$ is determining for a strongly harmonic function, see Section 6 for details. 
Our presentation is largely self contained and for the readers convenience in Sections 2 and 3 we recall the necessary definitions and observations regarding Carnot-Carathéodory groups, pseudonorms, subelliptic Laplacians and their fundamental solutions.

\section{Preliminaries}

In this section we recall some rudimentary properties of the geometry of CarnotCarathéodory groups (CC-groups or Carnot groups, for short). Upon recalling the definition of a CC-group, we illustrate the notions with examples of groups playing an important role in our studies, namely the $H$-type groups and the Heisenberg groups $\mathbb{H}_{n}$. Then in Section 2.2 we provide basic information about pseudonorms and pseudodistances. Definitions and results presented in that section will be used in our studies of the strongly harmonic functions, cf. Definition 3.2, and their relations to the $\mathcal{L}$-harmonic operator, see Section 4. Finally, in Section 2.3 we recall the notion of convolution and provide its properties needed in our further presentation.

\subsection{Carnot-Carathéodory Groups}

A Lie algebra $\mathfrak{g}$ is said to be stratified if $\mathfrak{g}=\mathfrak{g}_{1} \oplus \cdots \oplus \mathfrak{g}_{s}$, where the bracket generating property $\mathfrak{g}_{i+1}=\left[\mathfrak{g}_{1}, \mathfrak{g}_{i}\right]$ holds, and $\left[\mathfrak{g}_{1}, \mathfrak{g}_{s}\right]=\{0\}$. The group $G=\exp (\mathfrak{g})$ is also said to be stratified and we adopt the notation $\tau_{p}(q)=p q$ for the left translation of $q \in G$ by $p \in G$. The left translates of $\mathfrak{g}_{1}$ define the horizontal subbundle $\mathcal{H} \subset T G$, which can naturally be equipped with a left invariant positive definite inner product, defined by the left translation of a scalar product given on $\mathfrak{g}_{1}$. The bracket generating property ensures that $G$ is path connected by absolutely continuous curves with horizontal tangents at almost every point. The sub-Riemannian distance between two points, denoted by $d_{s}$, is then the infimum of lengths of all horizontal curves joining them.

The Carnot-Carathéodory distance on $G$, or $C C$-distance for short, relative to an orthonormal left invariant horizontal frame $\left\{X_{i}\right\}$, is constructed as follows: Every absolutely continuous horizontal curve $\gamma$ joining two points $p$ and $q$, can be normalised by a change of parameter so that $\gamma:[0, T] \rightarrow G$ and $\sum_{i}\left\langle\dot{\gamma}, X_{i}\right\rangle^{2} \leq 1$. The Carnot-Carathéodory distance between two points, denoted by $d_{c c}$, is the infimum of those $T>0$ such that there exists a normalised curve $\gamma:[0, T] \rightarrow G$ joining the two points.

The Carnot-Carathéodory metric is in fact the sub-Riemannian distance. Indeed the horizontal curves parametrised by the sub-Riemannian arc length form a subfamily of the normalised curves such that $T=\ell(\gamma)$, and so the sub-Riemannian distance is bounded below by the Carnot-Carathéodory distance. Let $\gamma_{n}:\left[0, T_{n}\right] \rightarrow G$ be a sequence of normalised curves such that $T_{n}$ converges to the $C C$-distance. Since

$$
d_{c c}(p, q) \leq d_{s}(p, q) \leq \ell\left(\gamma_{n}\right)=\int_{0}^{T_{n}}\left|\dot{\gamma}_{n}(t)\right| d t \leq T_{n},
$$

we must therefore have $d_{c c}(p, q)=d_{s}(p, q)$. We remark that the discussion above applies to the more general setting where $G$ is simply a manifold with a bracket generating subbundle of the tangent bundle.

Hence we will call a stratified Lie group $G$ with a sub-Riemannian distance a CarnotCarathéodory group. The normal model of $G$ is denoted $(\mathfrak{g}, *)$ where $*$ is given by the Baker-Campbell-Hausdorff formula, i.e., the exponential is an isomorphism. Dilation $\delta_{\lambda}$ of $\mathfrak{g}$ by $\lambda>0$ is given by $\delta_{\lambda}(X)=\sum_{i} \lambda^{i} X_{i}$ where $X_{i}$ is the projection of $X$ onto $\mathfrak{g}_{i}$. An 
immediate consequence of the definition is that $\delta_{\lambda} \in \operatorname{aut}(\mathfrak{g})$ (=the automorphism group of the Lie algebra). Conjugating $\delta_{\lambda}$ with the exponential map defines dilation of $G$ which is again in $\operatorname{aut}(G)$, since for the normal model we have aut $(G)$ and aut(g) are one and the same.

We illustrate the above discussion with two main examples of the Carnot-Carathéodory groups. In what follows we will frequently appeal to these examples and assume that the reader is familiar with them. In both cases, the orthonormal basis for $\mathfrak{g}_{1}$ is extended to $\mathfrak{g}$, then the associated scalar product defines a length on $\mathfrak{g}$, which we denote by $|X|$ for each $X \in \mathfrak{g}$.

Example 1 The $n$-dimensional Heisenberg group $G=\mathbb{H}_{n}$ is the Carnot group with a 2-step Lie algebra and orthonormal basis $\left\{X_{1}, \ldots, X_{2 n}, Z\right\}$ such that

$$
\mathfrak{g}_{1}=\operatorname{Span}\left\{X_{1}, \ldots, X_{2 n}\right\}, \quad \mathfrak{g}_{2}=\operatorname{Span}\{Z\}
$$

and the nontrivial brackets are $\left[X_{i}, X_{n+i}\right]=Z$ for $i=1, \ldots, n$.

In particular, if $n=1$, then a natural basis for the left invariant vector fields is given by the following vector fields:

$$
\tilde{X}=\frac{\partial}{\partial x}+2 y \frac{\partial}{\partial t}, \quad \tilde{Y}=\frac{\partial}{\partial y}-2 x \frac{\partial}{\partial t} \quad \text { and } \quad \tilde{T}=\frac{\partial}{\partial t},
$$

where $[\tilde{X}, \tilde{Y}]=-4 \tilde{T}$. Note that these fields are defined with respect to the multiplication given by $V * W=V+W-4[V, W]$ for $V, W \in \mathfrak{g}$, which is not the Baker-CampbellHausdorff formula. The reason for choosing this slightly less orthodox multiplication is that it leads to a simpler expression for the Folland-Kaplan pseudonorm derived from the fundamental solution of $\mathcal{L}=\tilde{X}^{2}+\tilde{Y}^{2}$ (see Example 4 below).

Example 2 An $H$-type group is a connected, simply connected 2-step Carnot group whose Lie algebra satisfies the following additional property: For each $Z \in \mathfrak{g}_{2}$ the homomorphism $J_{Z}: \mathfrak{g}_{1} \rightarrow \mathfrak{g}_{1}$ defined by

$$
\left\langle J_{Z} X, Y\right\rangle=\langle Z,[X, Y]\rangle, \quad \text { for all } X, Y \in \mathfrak{g}_{1}
$$

satisfies $J_{Z}^{2}=-\langle Z, Z\rangle I$.

Definition 2.1 (Change of Basis) A basis $\mathcal{E}$ of $\mathfrak{g}$ is said to be adapted, if it has the form

$$
\mathcal{E}=\left\{E_{1}^{1}, \ldots, E_{N_{1}}^{1}, \ldots, E_{1}^{s}, \ldots, E_{N_{s}}^{s}\right\},
$$

where $\mathfrak{g}_{k}=\operatorname{span}\left\{E_{1}^{k}, \ldots, E_{N_{k}}^{k}\right\}$. Moreover, we define $N:=N_{1}+N_{2}+\ldots+N_{s}$.

Note that if $\tilde{\mathcal{E}}$ is also adapted to $\mathfrak{g}$, and $A$ is the transition matrix defined by $\pi_{\mathcal{E}}(X)=$ $\pi_{\tilde{\mathcal{E}}}(A X)$ where $\pi_{\mathcal{E}}$ and $\pi_{\tilde{\mathcal{E}}}$ are the coordinate projections, then $A$ is a strata-preserving automorphism of $\mathfrak{g}$ and a strata-preserving isomorphism of $(\mathfrak{g}, *)$.

The left translates of $\mathfrak{g}_{1}$ define the horizontal subbundle $\mathcal{H} \subset T G$ which is naturally equipped with a left invariant sub-Riemannian metric $d_{s}$, defined by the left translation of the scalar product restricted to $\mathfrak{g}_{1}$. It is easy to see using normal coordinates, that a left Haar measure on $G$ is also a right Haar measure, and is simply the Lebesgue measure, up to scale, defined by the ambient Euclidean structure of the normal model. 
If we choose a basis $\left\{E_{1}^{1}, \ldots, E_{N_{1}}^{1}\right\}$ of $\mathfrak{g}_{1}$, then the left and right invariant vector fields corresponding to $E_{i}^{1}$ are defined as follows:

$$
\tilde{X}_{i}^{l} u(p)=\left.\frac{d}{d t} u\left(p e^{t E_{i}^{1}}\right)\right|_{t=0} \quad \tilde{X}_{i}^{r} u(p)=\left.\frac{d}{d t} u\left(e^{t E_{i}^{1}} p\right)\right|_{t=0} .
$$

The corresponding left and right invariant sub-Laplacians are

$$
\mathcal{L}^{L} u(p)=\sum_{i} \tilde{X}_{i}^{l} \tilde{X}_{i}^{l} u(p) \quad \mathcal{L}^{R} u(p)=\sum_{i} \tilde{X}_{i}^{r} \tilde{X}_{i}^{r} u(p) .
$$

We present our results in terms of left invariant fields and adopt the convention that unless otherwise stated, $\tilde{X}_{i}$ and $\mathcal{L}$ will be left invariant.

We emphasize the role of the sub-Laplacians among the sub-elliptic operators in the following remark.

Remark 1 Let us consider a generalized sub-Laplacian, namely a left invariant operator of the form:

$$
L_{A} u=\sum_{i j} a_{i j} \tilde{X}_{i} \tilde{X}_{j} u
$$

where $A=\left(a_{i j}\right)$ is a symmetric and positive definite matrix with constant real coefficients. Set $B:=A^{1 / 2}$, then by the bracket generating property, $B$ extends to a strata preserving automorphism of $\mathfrak{g}$ such that $L_{A}=\sum_{k} \tilde{Y}_{k}^{2}$, where $\tilde{Y}_{k}=\sum_{l} b_{k l} \tilde{X}_{l}$. In other words, operator (3) can be reduced to a sub-Laplacian by a strata preserving automorphism, see [3] page 64 for more details.

\subsection{Pseudonorm and Pseudodistance}

The following definitions and remarks are crucial for our considerations and are largely applied in Sections 3 and 4. For further studies of pseudonorms and pseudodistances we refer, for instance, to the book of Bonfiglioli-Lanconelli-Uguzzoni [3].

Definition 2.2 A continuous function $\mathcal{N}: G \rightarrow[0, \infty)$ is said to be a symmetric pseudonorm on a Carnot group $G$, if $\mathcal{N}$ satisfies the following conditions:

1. $\mathcal{N}\left(\delta_{r}(p)\right)=r \mathcal{N}(p)$ for every $r>0$ and $p \in G$,

2. $\mathcal{N}(p)>0$ if and only if $p \neq 0$,

3. $\mathcal{N}$ is symmetric, i.e., $\mathcal{N}\left(p^{-1}\right)=\mathcal{N}(p)$ for every $p \in G$.

All pseudonorms of interest here will be symmetric, and so their symmetry will not be emphasized in their reference.

Example 3 Let $G$ be a group expressed in coordinates by choosing an orthonormal basis of $\mathfrak{g}$. Let us further write any point $p \in G$ as $p=\sum_{i=1}^{s} P_{i}$, where $P_{i} \in \mathfrak{g}_{i}$. Then it follows that for every $\alpha \geq 1$, a function

$$
\mathcal{N}(p)=\left(\sum_{i=1}^{s}\left|P_{i}(p)\right|^{\alpha / i}\right)^{1 / \alpha}
$$


defines a pseudonorm on $G$. For an appropriate choice of $\alpha, \mathcal{N}$ is $C^{\infty}$ on $G \backslash\{0\}$, e.g., $\alpha=2 s !$.

Example 4 Let $G=\mathbb{H}_{1}$ be the first Heisenberg group. Then, the Folland-Kaplan pseudonorm derived from the fundamental solution of $\mathcal{L}=\tilde{X}^{2}+\tilde{Y}^{2}$ is given by

$$
\mathcal{N}(z, t)=\left(|z|^{4}+t^{2}\right)^{1 / 4},
$$

where coordinates of any point $p \in \mathbb{H}_{1}$ are $p=(z, t)$ with $z \in \mathbb{C}$ and $t \in \mathbb{R}$. It follows that the horizontal gradient of $\mathcal{N}$ (see Eq. 5 below) satisfies

$$
\left|\nabla_{0} \mathcal{N}(z, t)\right|^{2}=\frac{|z|^{2}}{\sqrt{|z|^{4}+t^{2}}} .
$$

It turns out that a pseudonorm defines a pseudodistance on a group.

Definition 2.3 We say that a left invariant pseudodistance is induced by a pseudonorm $\mathcal{N}$, if

$$
d(p, q):=\mathcal{N}\left(p^{-1} q\right), \quad \text { for any } p, q \in G .
$$

In particular:

(i) $d(p, q) \geq 0$ with equality if and only if $p=q$,

(ii) there exists a constant $c>0$, such that

$$
d\left(p_{1}, p_{2}\right) \leq c\left(d\left(p_{1}, p_{0}\right)+d\left(p_{0}, p_{2}\right)\right) \quad \text { for all } p_{0}, p_{1}, p_{2} \in G .
$$

Although pseudodistances are not metrics, they still define a reasonable family of sets that play the role of balls.

Definition 2.4 Let $d$ be a given pseudometric on $G$. The ball centered at $p \in G$, of radius $r$ with respect to $d$, is denoted $B(p, r)$. Since left translation are isometries and are 1homogeneous with respect to dilation, we have

$$
B(p, r)=p \delta_{r}(B(0,1)) .
$$

where $B(0,1)$ is the unit ball centered at the origin.

Note that it follows that the Lebesgue measure of the ball $B(p, r)$ satisfies $|B(p, r)|=$ $r^{Q}|B(0,1)|$, where $Q=\sum_{i} i \operatorname{dim} \mathfrak{g}_{i}$ is the Hausdorff dimension of $G$.

We finish the presentation of basic properties of pseudonorms and pseudodistances with the observation that all pseudonorms on a given group $G$ are equivalent.

Remark 2 (cf. Proposition 5.1.4 in [3]) For any pair of pseudo-norms $\mathcal{N}$ and $\mathcal{N}^{\prime}$ on $G$, there exists $c>0$ such that

$$
\frac{1}{c} \mathcal{N}(p) \leq \mathcal{N}^{\prime}(p) \leq c \mathcal{N}(p) .
$$

Moreover, if $d_{s}$ stands for a sub-Riemannian distance in $G$, then $\mathcal{N}^{\prime}(p)=d_{s}(0, p)$ is a pseudonorm and so $d$ and $d_{s}$ are equivalent pseudodistances.

\subsection{Convolution}

Below we recall the notion of the convolution and some of its integrability and regularity properties. The section is based on presentation in Chapter 1 in Folland-Stein [10]. 
Let $G$ be a stratified group. The convolution of two measurable functions $g$ and $h$ on $G$ is defined as follows:

$$
g * h(p)=\int_{G} g(q) h\left(q^{-1} p\right) d q=\int_{G} g\left(p q^{-1}\right) h(q) d q,
$$

provided the integrals converge. In contrast with Euclidean spaces, the convolution is not commutative. The basic properties are as follows, cf. Propositions 1.19, 1.20 and pg. 22 in [10]:

1. Let $1 \leq r, s, t<\infty$ such that $r^{-1}+s^{-1}=t^{-1}+1$. If $g \in L^{r}(G)$ and $h \in L^{s}(G)$, then $g * h \in L^{t}(G)$ and $\|g * h\|_{t} \leq c(r, s)\|g\|_{r}\|h\|_{s}$.

2. If $L$ is a left invariant vector field, then $L(g * h)=g *(L h)$ provided that $h$ is $C^{2}$ smooth.

3. If $R$ is a right invariant vector field, then $R(g * h)=(R g) * h$ provided that $g$ is $C^{2}$-smooth.

4. If $L$ and $R$ are corresponding left and right invariant vector fields (i.e., they agree at the identity), then $(L g) * h=g *(R h)$ when $g$ and $h$ are suitably smooth.

5. If $\psi \in L^{1}(G)$ and $\psi_{\epsilon}=\epsilon^{-Q} \psi \circ \delta_{1 / \epsilon}$, then $\int_{G} \psi_{\epsilon}(q) d q=\int_{G} \psi(q) d q$.

6. If $h \in L^{r}(G), 1 \leq r<\infty$ and $\int_{G} \psi(q) d q=c$, then $\left\|h * \psi_{\epsilon}-c h\right\|_{r} \rightarrow 0$ as $\epsilon \rightarrow 0$.

7. If $h$ is continuous and bounded on $\Omega$, then $h * \psi_{\epsilon} \rightarrow c h$ locally uniformly on $\Omega$ as $\epsilon \rightarrow 0$.

For the sake of completeness of the discussion, we further recall that convolutions can be defined also in the setting of distributions. Namely, we set $(\Lambda * g, \phi)=(\Lambda, \phi * \tilde{g})$ and $(g * \Lambda, \phi)=(\Lambda, \tilde{g} * \phi)$ for any $\phi \in C_{0}^{\infty}(G)$, where $\tilde{g}(p):=g\left(p^{-1}\right)$. Therefore, it then follows that if $\Lambda_{u}$ is a distribution defined as $\left(\Lambda_{u}, \phi\right)=\int_{G} u \phi$, then

$$
\left(\Lambda_{u} * g, \phi\right)=\left(\Lambda_{u * g}, \phi\right) \quad \text { and } \quad\left(g * \Lambda_{u}, \phi\right)=\left(\Lambda_{g * u}, \phi\right) .
$$

\section{Sub-Laplacians, Fundamental Solutions and Mean Value Property}

The first goal of this section is to recall the weak formulation of the sub-Laplacian together with a notion of the fundamental solution and its relation with pseudonorms. Then, in Section 3.2 we give the definition of strongly harmonic functions, one of the main objects studied in this work. Such functions are defined originally in $[1,11]$ in the setting of metric measure spaces.

We begin our discussion by recalling the horizontal Sobolev spaces and the weak formulation of the $\mathcal{L}$-harmonic equation, cf. Eqs. 2 and 3.

Let $\Omega \subset G$ be open. Recall that a $C^{2}(\Omega)$-smooth function $u$ is said to be $\mathcal{L}$-harmonic on $\Omega$, if $\mathcal{L} u(p)=0$ for all $p \in \Omega$, cf. Eq. 2. In order to weaken the $C^{2}$-regularity assumption one considers $\mathcal{L}$ on an appropriate Sobolev space.

Recall that $N_{1}$ denotes the dimension of $\mathfrak{g}_{1}$, cf. Definition 2.1. For $1<s<\infty$, we say that a function $u: \Omega \rightarrow \mathbb{R}$ belongs to the horizontal Sobolev space $H W^{1, s}(\Omega)$, if $u \in L^{s}(\Omega)$ and for all $i=1, \ldots, N_{1}$, the horizontal derivatives $\tilde{X}_{i} u$ exist in the distributional sense and are represented by elements of $L^{s}(\Omega)$. The space $H W^{1, s}(\Omega)$ is a Banach space with respect to the norm

$$
\|u\|_{H W^{1, s}(\Omega)}=\|u\|_{L^{s}(\Omega)}+\left\|\left(\tilde{X}_{1} u, \ldots, \tilde{X}_{N_{1}} u\right)\right\|_{L^{s}(\Omega)} .
$$

In the similar way we define the local spaces $H W_{l o c}^{1, s}(\Omega)$. Moreover, we define $H W_{0}^{1, s}(\Omega)$ as the closure of $C_{0}^{\infty}(\Omega)$ in the $H W^{1, s}(\Omega)$-norm. Recall, that $C_{0}^{\infty}(\Omega)$ stands for a set of 
smooth compactly supported functions in $\Omega$. The horizontal gradient $\nabla_{0} u$ of an element $u \in H W_{l o c}^{1, s}(\Omega)$ is defined by

$$
\nabla_{0} u=\sum_{i=1}^{N_{1}}\left(\tilde{X}_{i} u\right) \tilde{X}_{i} .
$$

If $u \in H W_{l o c}^{1,2}(\Omega)$, then $u$ is said to be weakly $\mathcal{L}$-harmonic on $\Omega$ if

$$
\left(\mathcal{L} \Lambda_{u}, \phi\right):=\int_{\Omega} u(q) \mathcal{L} \phi(q) d q=-\int_{\Omega}\left\langle\nabla_{0} u(q), \nabla_{0} \phi(q)\right\rangle d q=0
$$

for all $\phi \in C_{0}^{\infty}(\Omega)$.

\subsection{Fundamental Solutions}

The notion of the fundamental solution of the Laplace operator $L=\Delta$ is well known in the setting of domains in $\mathbb{R}^{n}$. Among many applications, fundamental solutions are used to solve the related Poisson equation $\Delta u=f$ via the Newtonian potentials of $f$ when $f$ is sufficiently regular, see e.g. Chapter 4 in Gilbarg-Trudinger [12] for the discussion of this classical topic.

Let us also recall the related notion of a hypoelliptic operator. We say that a differential operator $P$ with smooth coefficients, defined on an open subset $\Omega \subset \mathbb{R}^{n}$ is called hypoelliptic, if for every distribution $u$ defined on an open subset $\Omega^{\prime} \subset \Omega$ satisfying $P u \in C^{\infty}\left(\Omega^{\prime}\right)$, it holds that also $u \in C^{\infty}\left(\Omega^{\prime}\right)$. The connection between the hypoellipticity and the fundamental solutions can be formulated as follows (see e.g. Hörmander [14]): Let $\left\{\tilde{Y}, \tilde{X}_{1}, \ldots, \tilde{X}_{m}\right\}$ be smooth vectors fields on $\Omega \subset \mathbb{R}^{n}$. Suppose

$$
\operatorname{rank} \operatorname{Lie}\left\{\tilde{Y}, \tilde{X}_{1}, \ldots, \tilde{X}_{m}\right\}(x)=n, \quad \text { for all } x \in \Omega \text {. }
$$

Then the operator

$$
L=\tilde{Y}+\sum_{j=1}^{m} \tilde{X}_{j}^{2}
$$

is $C^{\infty}(\Omega)$-hypoelliptic. Thus, every distributional solution to $L u=f$ is represented by a function in $C^{\infty}(\Omega)$ when $f \in C^{\infty}(\Omega)$.

We move now our discussion of fundamental solutions to the setting of CC-groups.

Definition 3.1 (Fundamental solution) Let $G$ be a stratified Lie group and let $\mathcal{L}$ be a subLaplacian on $G$. A function $\Gamma: G \backslash\{0\} \rightarrow \mathbb{R}$ is called a fundamental solution for $\mathcal{L}$ if:

(i) $\Gamma \in C^{\infty}(G \backslash\{0\})$,

(ii) $\Gamma \in L_{\text {loc }}^{1}(G)$ and $\Gamma(q) \rightarrow 0$, when $q \rightarrow \infty$,

(iii) $\mathcal{L} \Gamma=\operatorname{Dirac}_{0}$, where Dirac 0 is the Dirac measure supported on $\{0\}$. More explicitly

$$
\int_{G} \Gamma(q) \mathcal{L} \phi(q) d q=\phi(0), \quad \text { for all } \phi \in C_{0}^{\infty}(G) .
$$

Note that in item (iii) we have chosen the convention $\mathcal{L} \Gamma=$ Dirac $_{0}$, where as some texts, e.g., [3], use the covention $\mathcal{L} \Gamma=-$ Dirac $_{0}$. The effect is merely a change of sign on $\Gamma$. In the latter case, $\Gamma$ is strictly positive on $G \backslash\{0\}$, see Proposition 5.3.13 in [3], whereas in our convention $\Gamma$ is strictly negative on $G \backslash\{0\}$.

On the existence and uniqueness of fundamental solutions we have the following theorem. 
Theorem 3.1 (Theorem 2.1 in Folland [9], Theorem 5.3.2 and Proposition 5.3.10 in [3]) Let $\mathcal{L}$ be a sub-Laplacian on $G$. Then there exists a unique fundamental solution $\Gamma$ for $\mathcal{L}$.

The relation between the $\mathcal{L}$-harmonicity and the pseudodistances plays important role in our discussion, see Section 4. For this reason, we now recall, following Definition 5.4.1 in [3], that a homogeneous symmetric pseudonorm $\mathcal{N}$ on $G$, is called an $\mathcal{L}-$ gauge if

$$
\mathcal{L}\left(\mathcal{N}^{2-Q}\right)=0 \quad \text { in } G \backslash\{0\} .
$$

The fundamental solution defines an $\mathcal{L}-$ gauge:

Theorem 3.2 (Proposition 5.4.2 and Theorem 5.5.6 in [3]) Let $\Gamma$ be the fundamental solution of a sub-Laplacian $\mathcal{L}$ defined on a group $G$ of homogeneous dimension $Q>2$. Then,

$$
\mathcal{N}(p)= \begin{cases}(-\Gamma)^{1 /(2-Q)}(p) & p \in G \backslash\{0\}, \\ 0 & p=0\end{cases}
$$

is an $\mathcal{L}$-gauge and defines a pseudodistance (see Definition 2.3). Conversely, any $\mathcal{L}$-gauge $\mathcal{N}$ satisfies $\Gamma=C \mathcal{N}^{2-Q}$ for some constant $C$.

We illustrate the above theorems with an example of the $H$-type groups.

Example 5 Basing on Example 2, we recall that a Lie algebra $\mathfrak{g}$ of an $H$-type group decomposes as follows: $\mathfrak{g}=\mathfrak{g}_{1} \oplus \mathfrak{g}_{2}$. If $\pi_{i}: \mathfrak{g} \rightarrow \mathfrak{g}_{i}, i=1,2$, are the natural projections, then the Folland-Kaplan pseudonorm on the normal model $(\mathfrak{g}, *)$ is given by the following formula:

$$
\mathcal{N}(X):=a(X)^{\frac{1}{4}}:=\left(\left\langle\pi_{1}(X), \pi_{1}(X)\right\rangle^{2}+16\left\langle\pi_{2}(X), \pi_{2}(X)\right\rangle\right)^{\frac{1}{4}},
$$

and so the fundamental solution for the $\mathcal{L}$-Laplace operator takes a form:

$$
-\Gamma(X)=c \mathcal{N}(X)^{2-Q},
$$

where $c=c(Q)>0$.

Finally, we recall a representation formula for test functions in terms of the subLaplacian, see Theorem 5.3.3 in [3]. Namely, by applying Formula (6) to a test function $G \ni q \rightarrow \phi(p q)$ defined for any given $\phi \in C_{0}^{\infty}(G)$ and any $p \in G$, gives the following:

$$
\int_{G} \Gamma\left(p^{-1} q\right) \mathcal{L} \phi(q) d q=-\phi(p),
$$

where $\Gamma$ is a fundamental solution for $\mathcal{L}$ in $G$. A similar formula arises in the studies of the non-homogeneous sub-Laplace equation $\mathcal{L} u=f$ with $f \in C_{0}^{\infty}(G)$. Then, it holds:

$$
u(p)=f * \Gamma(p)=\int_{G} f(q) \Gamma\left(q^{-1} p\right) d q .
$$

For further studies about this equation and the associated linear potentials we refer, for instance, to the book of Ricciotti [18].

\subsection{Mean Value Property - Strongly and Weakly Harmonic Functions}

In the previous sections we recalled and discussed the harmonicity from the point of view of the subelliptic Laplacian $\mathcal{L}$. Below we introduce a different approach based on the 
mean value property, the so-called strongly and weakly harmonic functions. Such functions are defined in the general setting of metric measure spaces, and were introduced in Gaczkowski-Górka [11] and studied further in [1], where several rudimentary properties of such functions are proved. These results include the maximum and comparison principles, various types of Harnack estimates, local Hölder and Lipschitz regularity, the Liouville type theorems and the solvability of the Dirichlet problem based on the dynamical programming method and the Perron method. The interplay between the underlying measure and metric turns out to be crucial in such studies. Furthermore, the flexibility in choosing the distance function and measures sheds new light on harmonic functions.

Following the discussion in Section 3 in [1], we recall the key definitions specialized to the setting of Carnot-Carathéodory groups. In this section, by $d$, we denote a metric induced by a left-invariant pseudonorm $\mathcal{N}$ on a Carnot-Carathéodory group $G$, cf. Section 2.2. A ball $B(p, r)$ in $G$ centered at $p \in G$ with radius $r>0$ is defined as follows:

$$
B(p, r)=\{q \in G: d(p, q) \leq r\} .
$$

Definition 3.2 Let $\Omega \subset G$ be an open set. A locally integrable function $f: \Omega \rightarrow \mathbb{R}$ is called (strongly) harmonic in $\Omega$ if the following inequality holds for all balls $B(p, r) \Subset \Omega$ with $p \in \Omega$ and $r>0$ :

$$
f(p)=\frac{1}{|B(p, r)|} \int_{B(p, r)} f(q) d q .
$$

Here, $d q$ stands for the Lebesgue measure. The set of all harmonic functions in $\Omega$ will be denoted $\mathcal{H}(\Omega)$.

Let us comment that in general the notion of strongly harmonic functions depends on the underlying measure and the distance (see [1]). In what follows we consider the Lebesgue measure only, but the balls are considered with respect to various different metrics. We further remark that a similar notion of harmonicity has been considered in the probabilistic setting in the context of the large-scale analysis on metric measure spaces, see Section 2.1 and Remark 2.5 in Tessera [19].

The following definition generalizes the previous one and strengthens the motivation for studying harmonic functions defined via the mean value property, see the discussion below.

Definition 3.3 Let $\Omega \subset G$ be an open set. A locally integrable function $f: \Omega \rightarrow \mathbb{R}$ is called weakly harmonic in $\Omega$ if for every $p \in \Omega$ there exists a non-empty set of positive radii $\left\{r_{p, \alpha}\right\}_{\alpha \in I}$, for some indexing set $I$, such that the following inequality holds for all balls $B\left(p, r_{p, \alpha}\right) \Subset \Omega$ :

$$
f(p)=\frac{1}{\left|B\left(p, r_{p, \alpha}\right)\right|} \int_{B\left(p, r_{p, \alpha}\right)} f(q) d q .
$$

As in the previous definition, $d q$ denotes the Lebesgue measure. The set of all weakly harmonic functions in $\Omega$ will be denoted $w \mathcal{H}(\Omega)$.

Let us also remark that the above definitions are robust enough to allow us to study the mean value property not only for various measures and distances but in fact for pseudodistances as presented in Section 4.2 below. Indeed, it turns out that the $C^{\infty}$-regularity of functions in $\mathcal{H}$ holds also if $d$ is a pseudodistance. 
In the Euclidean setting, the relations between harmonicity and mean value property has been a subject of research for two centuries, and it was Gauss who, perhaps first, showed that harmonic functions have the mean value property (see e.g. Chapter VII.6 in Kellogg [15]). The opposite question, whether all radii of balls contained in an underlying domain are needed for the mean value property to hold has also been investigated by several prominent mathematicians, such as Koebe, Volterra and Kellogg, Hansen and Nadirashvili, Blaschke, Privaloff and Zaremba. Let us also remark, that several results in this direction of studies are known as one radius (two radii) theorems. We refer to [1] for further historical comments and references regarding the mean value property and harmonicity. The mean value property continues to inspire studies in PDEs, leading for instance to results on probabilistic characterizations of harmonicity in the metric setting (see Chen [7]), also to $p$-harmonious functions and their stochastic tug-of-war games (see [1]).

\section{Regularity of Weakly and Strongly Harmonic Functions and Their Relations with $\mathcal{L}$-Harmonic Functions}

The main results of the paper are presented in this section. Among other topics, we study regularity properties of weakly and strongly harmonic functions. First we show that the familiar method of difference quotients applies to weakly and strongly harmonic functions and obtain the local Sobolev regularity of such functions, see Theorem 4.1. We then prove that functions satisfying Definition 3.2 in Carnot groups are smooth (see also Remark 4 commenting the lack of a similar property for weakly harmonic functions). This observation is then used in our studies of relations between the strongly harmonic functions and the canonical sub-Laplacian $\mathcal{L}$. Namely, by using Theorem 4.2, we show that strongly harmonic functions defined with respect to the pseudodistance induced by the fundamental solution for $\mathcal{L}$ (which in the particular example of the Heisenberg group is a metric) satisfy the $\mathcal{L}$-harmonic equation, i.e., they are $\mathcal{L}$-harmonic, see Theorem 4.3 .

We present both Sobolev and $C^{\infty}$-regularity properties of strongly harmonic functions. The reason for this is that techniques employed in these studies differ from each other. The Sobolev type regularity is based on the measure theoretic properties of functions in the class $\mathcal{H}$, while the smoothness result relies on their analytic properties and the structure of Carnot-Carathéodory groups, in particular with respect to convolutions. Furthermore, for the smoothness of strongly harmonic functions we may weaken the assumptions on the underlying distance and allow it to be merely a pseudodistance. This observation generalizes definitions presented in [1] and is possible here as the more general definition of a harmonic function is now compensated by the presence of group and Euclidean structures as in $\mathbb{H}_{n}$. Therefore, the results of Sections 4.1 and 4.2 illustrate the richness of the structure of functions in $\mathcal{H}$ and provide additional motivation for further investigation.

\subsection{Local Sobolev Regularity}

Let $Z$ be a vector field in a Carnot group $G$ and $u: \Omega \rightarrow \mathbb{R}$ be a function from a domain $\Omega \subset G$. The difference quotient of $u$ at $p \in \Omega$ is defined as follows:

$$
D_{h}^{Z}(p)=\frac{u\left(p e^{h Z}\right)-u(p)}{h}=\frac{u\left(p e^{\delta_{h}(Z)}\right)-u(p)}{h}, \quad \text { when } \quad Z \in \mathfrak{g}_{1} \quad \text { and } h \neq 0
$$


We mention that the second quotient above is the Pansu difference quotient and that in general the second equality is false when $Z \notin \mathfrak{g}_{1}$. Recall that for any $V \in \mathfrak{g}$, the Pansu derivative is given by

$$
P D f(p)(V)=\lim _{h \rightarrow 0} \frac{u\left(p e^{\delta_{h}(V)}\right)-u(p)}{h}
$$

and is in some sense the natural derivative to consider on Carnot groups. In Lemma 3.6 of Capogna-Cowling [6], it is shown that $Q$-harmonic functions are Pansu differentiable almost everywhere. Note here that $Q$ is the Hausdorff dimension of $G$ and $u$ is $Q$-harmonic if and only if

$$
\int_{\Omega}\left|\nabla_{0} u(x)\right|^{Q-2}\left\langle\nabla_{0} u(x), \nabla_{0} \phi(x)\right\rangle d x=0
$$

for every $\phi \in C_{0}^{\infty}(\Omega)$. Note that $\mathcal{L}$-harmonic functions are 2-harmonic.

The following result holds in the Carnot group setting similarly to the Euclidean setting, cf. Gilbarg-Trudinger [12, Chapter 7.11], Hörmander [14], Manfredi-Mingione [17], Capogna [4] and Ricciotti [18].

Lemma 4.1 (cf. Lemma 1 in [17]) Let $\Omega \subset \mathbb{H}_{n}$ be a domain and $\Omega^{\prime} \Subset \Omega$. Furthermore, let $\tilde{Z}$ be a left-invariant vector field corresponding to $Z \in \mathfrak{g}_{1}$ and let $u \in L_{\text {loc }}^{s}(\Omega)$ for $s>1$. If there exist constants $\sigma<\operatorname{dist}\left(\Omega^{\prime}, \partial \Omega\right)$ and $C>0$ such that

$$
\sup _{0<|h|<\sigma} \int_{\Omega^{\prime}}\left|D_{h}^{Z} u(q)\right|^{s} d q \leq C^{s},
$$

then $\tilde{Z} u \in L^{s}\left(\Omega^{\prime}\right)$ and $\|\tilde{Z} u\|_{L^{s}\left(\Omega^{\prime}\right)} \leq C$. Conversely, if $\tilde{Z} u \in L^{s}\left(\Omega^{\prime}\right)$, then for some $\sigma>0$ it holds that

$$
\sup _{0<|h|<\sigma} \int_{\Omega^{\prime}}\left|D_{h}^{Z} u(q)\right|^{s} d q \leq\left(2\|\tilde{Z} u\|_{L^{s}\left(\Omega^{\prime}\right)}\right)^{s} .
$$

Moreover, if the first assertion holds, then $D_{h}^{Z} u$ converges strongly to $\tilde{Z} u$ in $L^{s}\left(\Omega^{\prime}\right)$, as $h \rightarrow 0$.

For another proof of the lemma, we refer to [18, Theorem 2.11], where it is stated and proved for the Heisenberg group. However, one easily observes that the proof holds for any Carnot group as well. We use the lemma to show the following result on the Sobolev regularity of strongly harmonic functions. Recall that a metric space $M$ is called geodesic, if every pair of points in $M$ can be joined by a curve whose length equals the distance between the points.

Theorem 4.1 Let $\Omega \subset G$ be a domain and let $\Omega^{\prime} \Subset \Omega$ with dist $\left(\Omega^{\prime}, \partial \Omega\right)>0$. Suppose that $u$ is a harmonic function in $\mathcal{H}(\Omega, d q)$, where $d q$ stands for the Lebesgue measure on $G$ and the underlying metric $d$ is such that $G$ is a geodesic space in $d$. Let $X \in \mathfrak{g}_{1}$, then $\tilde{X} u \in L^{s}\left(\Omega^{\prime}\right)$ for all $s>1$ and $u \in H W^{1, s}\left(\Omega^{\prime}\right)$.

In particular, if $G=\mathbb{H}_{n}$, and $\tilde{X}_{i}$ for $i=1,2, \ldots, 2 n$ are the left-invariant horizontal vector fields, then $\tilde{X}_{i} u \in L^{s}\left(\Omega^{\prime}\right)$ for all $s>1$ (see also the discussion following Definition 2.1 and Example 5).

In the proof below we appeal to a definition and some results from the general theory of strongly harmonic functions on metric measure spaces, developed in [1]. For the readers convenience we state them now. 
Let $(X, d, \mu)$ be a metric measure space. We say that a measure $\mu$ is continuous with respect to a metric $d$ if for all $x \in X$ and all $r>0$ it holds that

$$
\lim _{\Omega \ni y \rightarrow x} \mu(B(x, r) \Delta B(y, r))=0,
$$

where $\Delta$ denotes the symmetric difference of two sets.

Proposition 4.1 (a) Let $(X, d, \mu)$ be a geodesic doubling metric measure space. Then $\mu$ is continuous with respect to the metric d (see Proposition 2.1, [1]).

(b) Let $(X, d, \mu)$ be a metric measure space with measure $\mu$ continuous with respect to the metric d. If $f \in \mathcal{H}(\Omega, \mu)$, then $f$ is locally bounded in $\Omega$. (Corollary 4.1 in [1]).

Proof of Theorem 4.1 The proof appeals to the method presented, for instance, in the proof of Proposition 4.1 in [1]. Let us first observe that since $(G, d, d q)$ is, by its properties, a geodesic doubling space, then by Proposition 4.1(a), $\mu=d q$ is continuous with respect to the sub-Riemmanian metric and therefore Proposition 4.1(b) implies the local boundedness of $u$ in $\Omega$.

Let $h \in \mathbb{R}$ be such that $|h|<\operatorname{dist}\left(\Omega^{\prime}, \partial \Omega\right)$. Note that since $X \in \mathfrak{g}_{1}$ we have

$$
d\left(p, p e^{h X}\right)=d\left(p, e^{\delta_{h}(X)}\right)=|h| \mathcal{N}(X),
$$

where the norm $\mathcal{N}$ is such that $d(p, q)=\mathcal{N}\left(p^{-1} q\right)$ for all $p, q \in G$. The invariance of the measure implies that for any nontrivial $X \in \mathfrak{g}_{1}$, and any $r>0$ such that $B(p, r) \Subset \Omega^{\prime}$ and any $|h|<r / \mathcal{N}(X)$, we have

$$
\begin{aligned}
\left|u\left(p e^{h X}\right)-u(p)\right|= & \left|\frac{1}{\left|B\left(p e^{h X}, r\right)\right|} \int_{B\left(p e^{h X}, r\right)} u(q) d q-\frac{1}{|B(p, r)|} \int_{B(p, r)} u(q) d q\right| \\
= & \mid \frac{1}{\left|B\left(p e^{h X}, r\right)\right|} \int_{B\left(p e^{h X}, r\right)} u(q) d q-\frac{1}{\left|B\left(p e^{h X}, r\right)\right|} \int_{B(p, r)} u(q) d q \\
& -\frac{\left|B\left(p e^{h X}, r\right)\right|-|B(p, r)|}{\left|B\left(p e^{h X}, r\right)\right||B(p, r)|} \int_{B(p, r)} u(q) d q \mid \\
\leq & \frac{1}{\left|B\left(p e^{h X}, r\right)\right|} \int_{B\left(p e^{h X}, r\right) \Delta B(p, r)}|u(q)| d q \\
& +\frac{|| B\left(p e^{h X}, r\right)|-| B(p, r)||}{\left|B\left(p e^{h X}, r\right)\right||B(p, r)|}\|u\|_{L^{1}(B(p, r))} \\
\leq & \frac{\left|B\left(p e^{h X}, r\right) \triangle B(p, r)\right|}{\left|B\left(p e^{h X}, r\right)\right|}\|u\|_{L^{\infty}\left(\Omega^{\prime}\right)}+\frac{|| B\left(p e^{h X}, r\right)|-| B(p, r)||}{\left|B\left(p e^{h X}, r\right)\right||B(p, r)|}\|u\|_{L^{1}\left(\Omega^{\prime}\right)} .
\end{aligned}
$$

In the last step we use the local boundedness, see Proposition 4.1(b), and the local integrability of $u$, see Definition 3.2. Hence,

$$
\begin{aligned}
& \int_{\Omega^{\prime}}\left|\frac{u\left(p e^{h X}\right)-u(p)}{h}\right|^{s} d q \leq 2^{s}\left|\Omega^{\prime}\right|\left(\|u\|_{L^{\infty}\left(\Omega^{\prime}\right)}^{s}+\|u\|_{L^{1}\left(\Omega^{\prime}\right)}^{s}\right) \\
& \times\left(\left|\frac{\left|B\left(p e^{h X}, r\right) \Delta B(p, r)\right|}{h\left|B\left(p e^{h X}, r\right)\right|}\right|^{s}+\left|\frac{|| B\left(p e^{h X}, r\right)|-| B(p, r)||}{h\left|B\left(p e^{h X}, r\right)\right||B(p, r)|}\right|\right) .
\end{aligned}
$$


Note that the left-invariance of the Lebesgue measure $d q$ implies that the second term above vanishes. Moreover, since $d\left(p, p e^{h X}\right)=|h| \mathcal{N}(X)$, then for sufficiently small $|h|$ we have:

$$
\left|B\left(p e^{h X}, r\right) \Delta B(p, r)\right| \leq\left|B\left(p e^{h X}, r+|h| \mathcal{N}(X)\right) \backslash B(p, r-|h| \mathcal{N}(X))\right| .
$$

In a consequence, we obtain

$$
\left|\frac{\left|B\left(p e^{h X}, r\right) \triangle B(p, r)\right|}{h\left|B\left(p e^{h X}, r\right)\right|}\right| \leq\left|\frac{(r+|h| \mathcal{N}(X))^{Q}-(r-|h| \mathcal{N}(X))^{Q}}{h r^{Q}}\right| \leq 2 Q \frac{(r+|h|)^{Q-1}}{r^{Q}} \leq 2 Q \frac{3^{Q-1}}{r},
$$

where in the second estimate we use the mean value theorem applied to function $t$, for $t \in R_{+}$on the interval $[r-|h| \mathcal{N}(X), r+|h| \mathcal{N}(X)]$. We choose radii of balls above such that $r=\frac{1}{2} \operatorname{dist}\left(\Omega^{\prime}, \partial \Omega\right)$. Therefore, for sufficiently small $|h|$, we have the following estimate:

$$
\begin{aligned}
\int_{\Omega^{\prime}}\left|\frac{u\left(p e^{h X}\right)-u(p)}{h}\right|^{s} d q & \leq 4^{s} Q^{s}\left|\Omega^{\prime}\right|\left(\|u\|_{L^{\infty}\left(\Omega^{\prime}\right)}^{s}+\|u\|_{L^{1}\left(\Omega^{\prime}\right)}^{s}\right)\left(\frac{(r+|h|)^{Q-1}}{r Q}\right)^{s} \\
& \leq\left(2 Q 3^{Q-1}\right)^{s} \frac{\left(1+\left|\Omega^{\prime}\right|^{s}\right)\left|\Omega^{\prime}\right|\|u\|_{L^{\infty}\left(\Omega^{\prime}\right)}^{s}}{\operatorname{dist}^{s}\left(\Omega^{\prime}, \partial \Omega\right)} .
\end{aligned}
$$

Lemma 4.1 implies that $D_{h}^{X} u \rightarrow \tilde{X}(u)$ in $L^{s}\left(\Omega^{\prime}\right)$.

In particular, if $G=\mathbb{H}_{n}$, then the same convergence holds for $X_{i}$ with $i=1,2, \ldots, 2 n$. The proof is completed.

Remark 3 The assertion of Theorem 4.1 holds as well if $u$ is weakly harmonic function in $w \mathcal{H}(\Omega, d q)$, provided that for any $p \in \Omega^{\prime}$ the mean value property holds for $u$ with respect to the ball $B\left(p, \frac{1}{2} \operatorname{dist}\left(\Omega^{\prime}, \partial \Omega\right)\right)$. Indeed, if $u$ is weakly harmonic in $\Omega$, then all the estimates in the above proof hold provided that at the beginning of the proof we set $r=\frac{1}{2} \operatorname{dist}\left(\Omega^{\prime}, \partial \Omega\right)$.

\subsection{Strongly Harmonic Functions on Carnot-Carathéodory Groups are Smooth}

The purpose of the following discussion is to show that the geometric definition of strongly harmonic functions, cf. Definition 3.2, implies in the setting of Carnot-Carathéodory groups that such functions are $C^{\infty}$ smooth. In fact, we can prove this assertion for a wider class of functions, defined with respect to pseudodistances instead of metrics. Namely, suppose that $G$ is equipped with a pseudonorm $\mathcal{N}$ defining a pseudodistance $d$, cf. Definitions 2.2 and 2.3. Furthermore, let $\Omega \subset G$ be a domain and let $B(p, r) \Subset \Omega$ denote any ball defined with respect to $d$. We extend Definition 3.2 and call a function $u: \Omega \rightarrow R$ strongly harmonic if, under the above assumptions, $u$ satisfies Definition 3.2:

$$
u(p)=\frac{1}{|B(p, r)|} \int_{B(p, r)} f(q) d q, \quad \text { for all } p \in G .
$$

It turns out that strong harmonicity with respect to pseudoballs implies smoothness, see Theorem 4.2. This illustrates that our notion of strong harmonicity is robust and allows some degree of flexibility for the distance. Furthermore, we show that for the distance $d$ defining the fundamental solution of the $\mathcal{L}$-harmonic operator on $G$, strongly harmonic functions are a subset of $\mathcal{L}$-harmonic functions, see Theorem 4.3.

Before proving Theorem 4.2 we need to recall the following auxiliary lemma on the integration in the polar coordinates in $G$ and bump functions. 
Lemma 4.2 (cf. Proposition 1.25 in Folland-Stein [10]) Let $G$ be a Carnot group of the Hausdorff dimension $Q$. For any homogeneous norm $\mathcal{N}$ on $G$, there exists a unique Radon measure $d \sigma$ on the unit sphere $S(0,1)=\{q \in G: \mathcal{N}(q)=1\}$ giving the following polar coordinate expression of the integral:

$$
\int_{G} u(q) d q=\int_{0}^{\infty} \int_{S(0,1)} u\left(\delta_{r}(v)\right) r^{Q-1} d \sigma(v) d r .
$$

Next, for smoothing purposes we need some bump functions. If $\mathcal{N}$ is any fixed pseudonorm on $G$ and $k \in \mathbb{N}$, then the function $\psi: G \rightarrow \mathbb{R}$ defined by

$$
\psi(q)= \begin{cases}C e^{-\frac{1}{1-\mathcal{N}(q)^{k}}}, & \mathcal{N}(q)<1 \\ 0, & \mathcal{N}(q) \geq 1,\end{cases}
$$

where $C^{-1}=\int_{G} \psi(q) d q$, has the following properties:

(i) $\psi$ is continuous and compactly supported in $B(0,1)$,

(ii) $\quad \psi$ is $C^{\infty}$ on $G \backslash\{0\}$, when $\mathcal{N} \in C^{\infty}(G \backslash\{0\})$,

(iii) $\psi$ is constant on all spheres centered at 0 ,

(iv) $\psi$ is $C_{0}^{\infty}$, if $k$ can be chosen so that $\mathcal{N}^{k}$ is $C^{\infty}$ at 0 .

Note that item (iv) is fulfilled by the Folland-Kaplan pseudonorm with $k=4$ and the pseudonorms defined at Eq. 4 with $k=2 s$ !.

Alternatively, we can also use the bump function given by:

$$
\psi=\frac{1}{|B(0,1)|} \chi_{B(0,1)}
$$

In this case we define $\psi_{\epsilon}:=\epsilon^{-Q} \psi\left(\delta_{\frac{1}{\epsilon}}\right)$ and observe that

$$
u * \psi_{\epsilon}=\int_{B(p, \epsilon)} u(q) d q .
$$

Note that both $\psi$ defined in Eqs. 13 and 14 are constant on spheres, i.e., $\left.\psi \circ \delta_{r}\right|_{S(0,1)}$ is constant.

Theorem 4.2 Let $\mathcal{N}$ be any pseudo-norm such that Eq. 13 is a $C_{0}^{\infty}$ bump for an appropriate choice of $k \in \mathbb{N}$. If $u \in L_{\mathrm{loc}}^{1}(G)$, then

$$
u(p)=f_{B(p, R)} u(q) d q, \quad \text { for all } p \in G
$$

is equivalent to

$$
u(p) \int_{S(0,1)} d \sigma(v)=\int_{S(0,1)} u\left(p \delta_{r}(v)\right) d \sigma(v), \quad \text { for all } p \in G, \quad 0<r \leq R,
$$

where $B(p, R)$ and $S(p, R)$ are the balls and spheres, respectively, determined by $\mathcal{N}$. Furthermore, either of Eqs. 16 or 17 implies that $u \in C^{\infty}(G)$.

Proof The implication (17) $\Longrightarrow$ (16) follows immediately from Eq. 12. To check that also the opposite implication holds true, let us first observe that functions in $\mathcal{H}(G)$ are continuous in $G$. Indeed, Proposition 4.1 in [1] stays that continuity of measure $\mu$ with respect to the underlying metric $d$ implies that functions in $\mathcal{H}(\Omega, \mu, d)$ are continuous, see also Eq. 10. The proof of this observation relies on the estimate similar to the one in the beginning of 
Theorem 4.1. If $d$ is a pseudodistance determined by $\mathcal{N}$ instead of a distance, then we proceed as follows. Since group $G$ equipped with the Lebesgue measure $d q$ and the subRiemannian distance $d_{s}$ is geodesic and doubling, then by Proposition 4.1(a) we get that $d q$ is continuous with respect to $d_{s}$. Remark 2 enables us to conclude that $d q$ is continuous also with respect pseudodistance $d$ and hence, Proposition 4.1 in [1] applies to $d$ as well. Thus, we conclude continuity of $u$ in $G$.

Next, we observe that if Eq. 16 holds, then for $p \in G$ we have

$$
\int_{0}^{R}\left(u(p) \int_{S(0,1)} d \sigma(v)-\int_{S(0,1)} u\left(p \delta_{r}(v)\right) d \sigma(v)\right) r^{Q-1} d r=0
$$

and so it follows that

$$
\int_{R_{1}}^{R_{2}}\left(u(p) \int_{S(0,1)} d \sigma(v)-\int_{S(0,1)} u\left(p \delta_{r}(v)\right) d \sigma(v)\right) r^{Q-1} d r=0
$$

for all admissible $R_{1}$ and $R_{2}$ satisfying $0<R_{1}<R_{2}$. If $h(r)$ denotes the expression within the brackets in Eq. 18, then by the mean value theorem, we have $h(r) r^{Q-1}=0$ for all admissible $r$, and so $h(r)=0$ for all such $r$.

To check the converse, we first note that since $d q=d q^{-1}$, the mean value theorem also implies that

$$
\int_{S(0,1)} u\left(\delta_{r}(v)\right) d \sigma(v)=\int_{S(0,1)} u\left(\delta_{r}\left(v^{-1}\right)\right) d \sigma(v) .
$$

We are now in a position to show that Eq. 17 results in $u \in C^{\infty}$. Let $\psi$ be the $C_{0}^{\infty}$ bump given by Eq. 13 and let $u \in L_{\mathrm{loc}}^{1}(G)$. Then

$$
\begin{aligned}
u * \psi_{\epsilon}(p) & =\int_{G} u\left(p q^{-1}\right) \psi_{\epsilon}(q) d q \\
& =\int_{B(0, \epsilon)} u\left(p q^{-1}\right) \psi\left(\delta_{1 / \epsilon}(q)\right) \frac{1}{\epsilon} d q \\
& =\int_{B(0,1)} u\left(p \delta_{\epsilon}\left(w^{-1}\right)\right) \psi(w) d w \quad\left(w:=\delta_{1 / \epsilon}(q)\right) \\
& =\int_{0}^{1} \int_{S(0,1)} u\left(p \delta_{\epsilon r}\left(v^{-1}\right)\right) \psi\left(\delta_{r}(v)\right) r^{Q-1} d \sigma(v) d r \quad\left(v:=\delta_{1 / r}(w)\right) \\
& =\int_{0}^{1} \int_{S(0,1)} u\left(p \delta_{\epsilon r}\left(v^{-1}\right)\right) d \sigma(v) \eta(r) r^{Q-1} d r \quad\left(\eta(r):=\psi\left(\delta_{r}(v)\right)\right) \\
& =\int_{0}^{1} \int_{S(0,1)} u\left(p \delta_{\epsilon r}(v)\right) d \sigma(v) \eta(r) r^{Q-1} d r \\
& =u(p) \int_{0}^{1} \int_{S(0,1)} \eta(r) r^{Q-1} d \sigma(v) d r . \quad \text { (by (17)). }
\end{aligned}
$$

Furthermore, we also have that

$$
\begin{aligned}
1=\int_{B(0,1)} \psi(q) d q & =\int_{0}^{1} \int_{S(0,1)} \psi\left(\delta_{r}(v)\right) r^{Q-1} d \sigma(v) d r \\
& =\int_{0}^{1} \int_{S(0,1)} \eta(r) r^{Q-1} d \sigma(v) d r .
\end{aligned}
$$

Hence, it follows from Eqs. 19 and 20 that for any $p \in G$

$$
u * \psi_{\epsilon}(p)=u(p) \text {. }
$$


This, by the smoothing properties of convolutions, implies that $u$ is $C^{\infty}$ and completes the proof of the theorem.

Remark 4 Let us observe that the technique used in the above proof does not give the $C^{\infty}$ regularity for weakly harmonic functions. Indeed, the use of convolutions $u * \psi_{\epsilon}$ requires the mean value property to hold for all radii in a given ball, a property that fails in general for functions satisfying Definition 3.3.

\subsection{Strongly Harmonic Functions are $\mathcal{L}$-Harmonic}

Recall, that in this section we allow $d$ in Definition 3.2 to be any pseudodistance. In the proof we appeal to the $C^{\infty}$-regularity of strongly harmonic functions, cf. Theorem 4.2. However in fact $C^{2}$-regularity is enough for the result to hold. Recall from the definition that an $\mathcal{L}$-gauge $\mathcal{N}$ is a pseudonorm derived from the fundamental solution of the operator $\mathcal{L}$ (see Formula (7)).

Theorem 4.3 Let $\Omega$ be a domain in a Carnot-Carathéodory group $G$ with the Hausdorff dimension $Q$. Let further $u: \Omega \rightarrow \mathbb{R}$ be strongly harmonic with respect to the balls given by an $\mathcal{L}$-gauge $\mathcal{N}$. Then $u$ is $\mathcal{L}$-harmonic.

Proof By the definition, $u \in L_{l o c}^{1}(\Omega)$ and satisfies the mean-value property at every point $p \in \Omega$ and any ball $B(p, r) \Subset \Omega$. Let $d$ stand for a pseudometric defined by an $\mathcal{L}$-gauge $\mathcal{N}$ as in Definition 2.3. For any $s>0$ let us denote by $\Omega_{s}:=\{p \in \Omega: d(p, \partial \Omega)>s\}$. Set

$$
g_{t}:=\frac{1}{|B(0, t)|} \chi_{B(0, t)}
$$

Then, formulas (11) and (15) imply that for all admissible radii $0<r<s$ (i.e. such that $B(p, s) \Subset \Omega)$ we have the following equation:

$$
u * g_{r}(p)=u * g_{s}(p)
$$

for all $p \in \Omega_{s}$.

Claim: Let $\mathcal{L}^{R}$ be the right-invariant Laplacian corresponding to $\mathcal{L}$, cf. Eq. 2. Then for any $0<r<s$, the non-homogeneous equation

$$
\mathcal{L}^{R} w_{r, s}=g_{s}-g_{r}
$$

admits a $C^{1}$ solution with compact support in $B(0, s)$. In order to prove the claim we adopt the proof of Lemma 4.1 in Gilbarg-Trudinger [12] and refer to Theorem 4.5 in Ricciotti [18] on the growth estimates for the fundamental solution and its horizontal gradient. Since both the result and the employed techniques are classical we restrict our discussion to sketch only.

Let $\Gamma^{R}$ be a fundamental solution of $\mathcal{L}^{R}$ as above (cf. Theorem 3.1). We define

$$
\begin{aligned}
w_{r, s}(p) & :=\int_{\Omega} \Gamma^{R}\left(p q^{-1}\right)\left(g_{s}-g_{r}\right)(q) d q \\
& =\frac{1}{|B(0, s)|} \int_{B(0, s) \backslash B(0, r)} \Gamma^{R}\left(p q^{-1}\right) d q+\left(\frac{1}{|B(0, s)|}-\frac{1}{|B(0, r)|}\right) \int_{B(0, r)} \Gamma^{R}\left(p q^{-1}\right) d q .
\end{aligned}
$$


Since $\Gamma^{R} \in L_{l o c}^{1}(G)$ we have $w_{r, s} \in L_{l o c}^{1}(\Omega)$. Notice also, that $B(0, s)=\operatorname{supp} w_{r, s}$. Moreover, since for any $s$ such that $0<r<s$, the set $\overline{\Omega_{s}}$ is compact, [18, Theorem 4.5] applies (cf. also Remark 6.18 in Capogna [5]). In particular, upon setting $\Gamma_{p}^{R}\left(q^{-1}\right):=$ $\Gamma^{R}\left(p q^{-1}\right)$ for any fixed $p \in B(0, s)$ inequality [18, (4.71)] gives us the following estimate for the horizontal gradient $\nabla_{0} \Gamma_{p}^{R}\left(q^{-1}\right)\left(\nabla_{\mathbb{H}, q^{-1}} \Gamma^{R}\right.$ in the notation of [18]):

$$
\left|\nabla_{0} \Gamma_{p}^{R}\left(q^{-1}\right)\right| \leq C \frac{d\left(p, q^{-1}\right)}{\left|B_{d\left(p, q^{-1}\right)}(p)\right|} .
$$

This estimate, the definition of $w_{r, s}$ in Eq. 23 and standard telescopic argument imply that $\nabla_{0} w_{r, s} \in L_{l o c}^{1}(\Omega)$. Indeed, let us fix any domain $\Omega^{\prime} \Subset G$ and observe that if $p \in B(0, s)$, then $B(0, s) \subset B(p, 2 s)$. Furthermore, recall that $d q=d q^{-1}$ and that domains $B(0, s)$ and $B(0, s) \backslash B(0, r)$ are symmetric with respect to the origin, hence both $q$ and $q^{-1}$ are contained in these domains. Therefore, for all $p \in \Omega^{\prime}$ we obtain the following estimate

$$
\begin{aligned}
& \left|\nabla_{0} w_{r, s}(p)\right| \\
& \leq \frac{1}{|B(0, s)|} \int_{B(0, s) \backslash B(0, r)}\left|\nabla_{0} \Gamma_{p}^{R}\left(q^{-1}\right)\right| d q+\left(\frac{1}{|B(0, s)|}-\frac{1}{|B(0, r)|}\right) \int_{B(0, r)}\left|\nabla_{0} \Gamma_{p}^{R}\left(q^{-1}\right)\right| d q \\
& =\frac{1}{|B(0, s)|} \int_{B(0, s) \backslash B(0, r)}\left|\nabla_{0} \Gamma_{p}^{R}(q)\right| d q+\left(\frac{1}{|B(0, s)|}-\frac{1}{|B(0, r)|}\right) \int_{B(0, r)}\left|\nabla_{0} \Gamma_{p}^{R}(q)\right| d q \\
& \leq \frac{1}{|B(0, s)|} \int_{B(0, s) \backslash B(0, r)} \frac{d(p, q)}{\left|B_{d(p, q)}(p)\right|} d q+\left(\frac{1}{|B(0, s)|}-\frac{1}{|B(0, r)|}\right) \int_{B(0, r)} \frac{d(p, q)}{\left|B_{d(p, q)}(p)\right|} d q \\
& \leq \frac{1}{|B(0,1)|}\left(\frac{2}{s^{Q}}+\frac{1}{r^{Q}}\right) \int_{B(0, s)} \frac{d(p, q)}{\left|B_{d(p, q)}(p)\right|} d q \\
& \leq \frac{1}{|B(0,1)|}\left(\frac{2}{s^{Q}}+\frac{1}{r^{Q}}\right) \int_{B(p, 2 s)} \frac{d(p, q)}{\left|B_{d(p, q)}(p)\right|} d q \\
& \leq \frac{1}{|B(0,1)|}\left(\frac{2}{s^{Q}}+\frac{1}{r^{Q}}\right) \sum_{k=-1}^{\infty} \frac{s}{2^{k}} \int_{B\left(p, \frac{s}{2^{k}}\right) \backslash B\left(p, \frac{s}{2^{k+1}}\right)}^{\left|B_{d(p, q)}(p)\right|} d q \\
& \leq \frac{1}{|B(0,1)|}\left(\frac{2}{s^{Q}}+\frac{1}{r^{Q}}\right) \sum_{k=-1}^{\infty} \frac{s}{2^{k}} \frac{\left|B\left(p, \frac{s}{2^{k}}\right)\right|}{\left|B\left(p, \frac{s}{2^{k+1}}\right)\right|} \\
& \leq \frac{8 C s}{|B(0,1)|}\left(\frac{2}{s^{Q}}+\frac{1}{r^{Q}}\right)
\end{aligned}
$$

where in order to obtain the last estimate we employ the doubling property of the measure (constant $C>0$ depends on the doubling constant). From this we infer that

$$
\int_{B(0, s)}\left|\nabla_{0} w_{r, s}(p)\right| d p \leq 4 C\left(s+\left(\frac{s}{r}\right)^{Q}\right) .
$$

Hence, it follows that $\left|\nabla_{0} w_{r, s}\right| \in L_{l o c}^{1}(G)$ since the domain $\Omega^{\prime}$ is arbitrary. In particular, if $r=s / 2$ the $L_{l o c}^{1}$-estimate is uniform in $s$ for $s \rightarrow 0^{+}$. We appeal to this observation later in this proof. 
In fact, as we now show, $w_{r, s} \in C^{1}(\Omega)$. This discussion allows us to compute $\mathcal{L}^{R}\left(w_{r, s}\right)$ in a weak sense and obtain that $w_{r, s}$ is a solution to Eq. 22. By employing the reasoning similar to [12, Lemma 4.1], for any $\epsilon>0$ we define

$$
w_{\epsilon}(p)=\int_{\Omega} \Gamma^{R}\left(p^{-1} q\right)\left(g_{s}-g_{r}\right) \eta\left(\frac{\mathcal{N}\left(p^{-1} q\right)}{\epsilon}\right) d q,
$$

where $\eta \in C^{1}(\mathbb{R}), 0 \leq \eta \leq 1,0 \leq \eta^{\prime} \leq 2$ and $\eta \equiv 0$ for all $t \leq 1$ whereas for all $t \geq 2$ we require that $\eta \equiv 1$. Similarly to the Euclidean case one proves that $w_{\epsilon} \in C^{1}(G)$ and, furthermore, that

$$
w_{\epsilon} \rightarrow w_{r, s} \quad \text { and } \quad \nabla_{0} w_{\epsilon} \rightarrow \int_{\Omega}\left(\nabla_{0} \Gamma^{R}\left(p^{-1} q\right)\right)\left(g_{s}-g_{r}\right) d q,
$$

as $\epsilon \rightarrow 0$ uniformly on compacta in $\Omega$. This completes the proof of the claim.

It now follows from Property 4 in Section 2.3 and Eq. 21 that

$$
(\mathcal{L} u) * w_{r, s}=u * \mathcal{L}^{R} w_{r, s}=u * g_{s}-u * g_{r}=0
$$

for all $0<r<s$. Next we show that $\mathcal{L} u=0$ on $\Omega$. In order to complete this goal, denote $\phi_{s}:=-w_{s / 2, s}$. Then

$$
\mathcal{L}^{R}\left(s^{Q-2} \phi_{s} \circ \delta_{s}\right)=\frac{1}{|B(0,1)|}\left(\left(2^{Q}-1\right) \chi_{B(0,1 / 2)}-\chi_{B(0,1) \backslash B(0,1 / 2)}\right)=\mathcal{L}^{R}\left(\phi_{1}\right) .
$$

A similar argument to that in Proposition 5.3.12 of [3] shows that

$$
\Gamma^{R}\left(\delta_{s}\left(p q^{-1}\right)\right)=s^{2-Q} \Gamma^{R}\left(p q^{-1}\right),
$$

and so the following identity holds:

$$
\begin{aligned}
& \int_{G} \phi_{s}(p) d p \\
& =\frac{1}{s^{Q-2}} \int_{G} s^{Q-2} \phi_{s} \circ \delta_{s}(p) s^{Q} d p \\
& =s^{2} \int_{B(0, s)} s^{Q-2}\left[\frac{1}{|B(0, s)|} \int_{B(0, s) \backslash B(0, s / 2) \mid} \Gamma^{R}\left(\delta_{s}(p) q^{-1}\right) d q\right. \\
& \left.+\left(\frac{1}{|B(0, s)|}-\frac{1}{|B(0, s / 2)|}\right) \int_{B(0, s / 2)} \Gamma^{R}\left(\delta_{s}(p) q^{-1}\right) d q\right] d p \\
& =s^{2} \int_{B(0, s)} s^{Q-2}\left[\frac{1}{|B(0,1)|} \int_{B(0,1) \backslash B(0,1 / 2)} \Gamma^{R}\left(\delta_{s}\left(p q^{-1}\right)\right) d q\right. \\
& =s^{2} \int_{B(0, s)}\left[\frac{1}{|B(0,1)|} \int_{B(0,1) \backslash B(0,1 / 2)} \Gamma^{R}\left(p q^{-1}\right) d q\right. \\
& +\left(\frac{1}{|B(0,1)|}-\frac{1}{|B(0,1 / 2)|} \int_{B(0,1 / 2)} \Gamma^{R}\left(\delta_{s}\left(p q^{-1}\right)\right) d q\right] d p \\
& =s^{2} \int_{G} \phi_{1}(p) d p .
\end{aligned}
$$


Let us denote $c:=\int_{G} \phi_{1}(q) d q$ and let $\Omega^{\prime} \subset \Omega$ be compact. We define the following function:

$$
F^{q}(p)= \begin{cases}\mathcal{L} u\left(p q^{-1}\right), & \text { for } p \in \Omega^{\prime} q \\ 0, & \text { otherwise }\end{cases}
$$

and

$$
F(p)= \begin{cases}\mathcal{L} u(p), & \text { for } p \in \Omega^{\prime} \\ 0, & \text { otherwise }\end{cases}
$$

With this notation, we have

$$
\begin{aligned}
F * \frac{1}{s^{2}} \phi_{s}(p)-F(p) \int_{G} \phi_{1}(q) d q & =\frac{1}{s^{2}} \int_{G}\left(F\left(p q^{-1}\right)-F(p)\right) \phi_{s}(q) d q \\
& =\frac{1}{s^{2}} \int_{G}\left(F^{q}(p)-F(p)\right) \phi_{s}(q) d q \\
& =\frac{1}{s^{2}} \int_{G}\left(F^{\delta_{s}(q)}(p)-F(p)\right) \phi_{s} \circ \delta_{s}(q) s^{Q} d q \\
& =\int_{G}\left(F^{\delta_{s}(q)}(p)-F(p)\right) \phi_{1}(q) d q .
\end{aligned}
$$

By the Minkowski inequality applied to a fixed value of $1 \leq \alpha<\infty$ we get the following estimate:

$$
\left\|F * \frac{1}{s^{2}} \phi_{s}-c F\right\|_{L^{\alpha}(G)} \leq \int_{G}\left\|F^{\delta_{s}(q)}-F\right\|_{L^{\alpha}(G)}\left|\phi_{1}(q)\right| d q .
$$

Recall that by Theorem 4.2 we have $u \in C^{\infty}(G)$. This together with the definition of $F$ allows us to infer that $F \in L^{\alpha}(G)$ for all $1 \leq \alpha<\infty$. Since for all $p \in G$ it holds that $\left.\left\|F^{\delta_{s}(q)}(p)-F(p)\right\|_{L^{\alpha}(G)} \leq 2 \| F(p)\right) \|_{L^{\alpha}(G)}$, the dominated convergence theorem implies that

$$
\lim _{s \rightarrow 0}\left\|F * \frac{1}{s^{2}} \phi_{s}-c F\right\|_{L^{\alpha}(G)}=0 .
$$

Since $F * \frac{1}{s^{2}} \phi_{s}(p)=0$ we conclude that $\mathcal{L} u=0$ a.e. in $\Omega^{\prime}$. Therefore, we conclude that $\mathcal{L} u=0$ on $\Omega$ as $\Omega^{\prime}$ is an arbitrary compact subset of $\Omega$.

Theorem 4.3 shows that in Carnot groups, the strongly harmonic functions are a subfamily of the $\mathcal{L}$-harmonic functions. The opposite relation does not hold in general as demonstrated in Example 6 below, where a spherical harmonic polynomial, by definition satisfying the $\mathcal{L}$-harmonic equation, is shown not to be strongly harmonic. We postpone this example till Section 5 and discuss the spherical harmonics in more detail there. Moreover, in Section 5 we identify a subclass of spherical harmonic polynomials in $\mathbb{H}_{1}$ which are strongly harmonic.

We close this section with a consequence of Theorem 4.3, the so-called three spheres theorem. This part of the presentation is based on [2]. There, we show several variants of three-spheres theorems for sub-elliptic equations in Carnot groups of Heisenberg-type ( $H$-type groups).

The classical Hadamard three-circles theorem in $\mathbb{R}^{2}$ asserts that given three concentric circles with radii $0<R_{1}<R<R_{2}$ and a subharmonic function $u$ in the plane, the maximum of $u$ over a circle with radius $R$ is a convex function of $\log R$, with coefficients depending on the ratios of $R_{1}, R$ and $R_{2}$. The three-circles theorem has been generalized in various settings, including subharmonic functions in $\mathbb{R}^{n}$ for $n>2$, higher-dimensional concentric surfaces (e.g. three-spheres theorems), more general linear and quasilinear elliptic equations, the heat equation (three-parabolas theorem) and coupled elliptic systems of equations, see [2] for further details and references. 
Let $G$ be an $H$-type group and $\Omega \subset G$. For a function $u: \Omega \rightarrow \mathbb{R}$ we define

$$
M(r)=\sup \{u(X): X \in \Omega, \mathcal{N}(X)=r\} .
$$

The following observation holds.

Corollary 4.1 Let $G$ be an $H$-type group and $\Omega \subset G$ be a domain containing the identity element of $G$. Assume that $u: \Omega \rightarrow \mathbb{R}$ is strongly harmonic in $\Omega$ with respect to the pseudodistance $d$ derived from a given $\mathcal{L}$-gauge $\mathcal{N}$. Moreover, let us consider three concentric spheres in the pseudodistance derived from $\mathcal{N}$, with radii $r_{1}<r<r_{2}$ contained in $\Omega$. Then

$$
M(r) \leq M\left(r_{1}\right) \frac{r^{2-Q}-r_{2}^{2-Q}}{r_{1}^{2-Q}-r_{2}^{2-Q}}+M\left(r_{2}\right) \frac{r_{1}^{2-Q}-r^{2-Q}}{r_{1}^{2-Q}-r_{2}^{2-Q}} .
$$

Equality holds if and only if $u(X) \equiv \phi(r)$, where $r=\mathcal{N}(X)$ and $\phi$ is the function on the right-hand side of Eq. 24.

The proof of the corollary is the direct consequence of Theorem 4.3 above and Theorem 4 in [2].

\subsection{The Converse to Mean Value Property}

In this section we consider the situation where a function obeys a mean value property with respect to the underlying Lebesgue (Hausdorff) measure for balls in a given pseudodistance $d$ (not necessarily derived from an $\mathcal{L}$-gauge), and consider if we can provide necessary and sufficient conditions for such a function to be sub(super)harmonic with respect to $\mathcal{L}$ ? We restrict our discussion to the setting of the first Heisenberg group $\mathbb{H}_{1}$ due to complexity of the corresponding computations in $\mathbb{H}_{n}$ for $n \geq 2$.

First, let us motivate our studies with the following theorem. The most important conclusion of this result, especially relevant from our point of view, is that $\mathcal{L}$-harmonic functions need not in general be strongly harmonic. We formulate Theorem 4.4 in a setting of CCgroups, even though in what follows we will need this result only for the case $G=\mathbb{H}_{1}$. This illustrates that a relation between $\mathcal{L}$-harmonicity and harmonic functions as in Definitions 3.2 and 3.3 is involved for all CC-groups and requires further studies, see also discussion in Section 5 .

Theorem 4.4 Let $G$ be a Carnot-Carathéodory group and $u: \Omega \rightarrow \mathbb{R}$ be an $\mathcal{L}$-harmonic function in a domain $\Omega \subset G$. Furthermore, let $\mathcal{N}$ be a pseudonorm defined by the fundamental solution of $\mathcal{L}, c f$. Eq. 8. Then, the following volume mean value property holds for all $p \in G$ and all balls $B(p, r) \Subset \Omega$ :

$$
u(p)=\frac{\int_{B(p, r)} u(q)\left|\nabla_{0} \mathcal{N}\left(p^{-1} q\right)\right|^{2} d q}{\int_{B(p, r)}\left|\nabla_{0} \mathcal{N}\left(p^{-1} q\right)\right|^{2} d q}=\frac{\int_{B(0, r)} u(p q)\left|\nabla_{0} \mathcal{N}(q)\right|^{2} d q}{\int_{B(0, r)}\left|\nabla_{0} \mathcal{N}(q)\right|^{2} d q} .
$$

In the Euclidean setting we have $\left|\nabla_{0} \mathcal{N}\right|^{2} \equiv 1$ and Eq. 25 reduces to the mean value over Euclidean balls. In fact it is known, see [3, Chapter 5], that if $\left|\nabla_{0} \mathcal{N}\right|^{2}$ is a constant then $G$ is commutative and thus the geometry is Euclidean. It follows that equivalence between $\mathcal{L}$ harmonicity and the strong harmonicity in noncommutative Carnot groups does not occur, see the discussion following the proof of Theorem 4.3 and Example 6 below.

The proof of Theorem 4.4 is based on, nowadays, classical techniques employed in the studies of the Carnot-Carathéodory groups. Nevertheless, in Appendix we present it 
for the sake of completeness of the presentation and in order to demonstrate some crucial differences between the Euclidean and CC-settings.

Let $\Omega \subset \mathbb{H}_{1} \backslash\{0\}$ and let $u \in \mathcal{H}(\Omega, \mu)$, where $\mu=d q$ is the 3-Lebesgue measure (equivalently the 3-Hausdorff measure, denoted $\mathcal{H}^{3}$ ). Moreover, we assume that $d$ is a metric on $\mathbb{H}_{1}$. Therefore, it holds that

$$
u(p)=\frac{1}{\left|B_{d}(p, r)\right|} \int_{B_{d}(p, r)} u(q) d q,
$$

for all $p \in \Omega$ and every $r>0$ such that $B_{d}(p, r) \Subset \Omega$. By Theorem 4.2 we know that $u \in C^{\infty}(\Omega)$. On the other hand, by Theorem 5.6.1 in [3] we have that a $C^{2}$ function satisfies the following mean value property with respect to the pseudodistance $d_{\mathcal{L}}$ derived from an $\mathcal{L}$-gauge $\mathcal{N}$ :

$$
\begin{aligned}
u(p)= & \frac{1}{r^{4} \underset{B_{d_{\mathcal{L}}}(0,1)}{\int}\left|\nabla_{0} \mathcal{N}\right|^{2} d q}\left[\int_{B_{d_{\mathcal{L}}}(p, r)} u(q)\left|\nabla_{0} \mathcal{N}\right|^{2}\left(p^{-1} q\right) d q\right. \\
& \left.-2 \int_{B_{d_{\mathcal{L}}}(p, r)} \int_{0}^{r} \varrho^{3}\left(\int_{B_{d_{\mathcal{L}}}(p, r)}\left(\frac{1}{d^{2}\left(p^{-1} q\right)}-\frac{1}{\varrho^{2}}\right) \mathcal{L} u(q) d q\right) d \varrho\right] .
\end{aligned}
$$

Observe that the first expression on the right-hand side of Eq. 27 is the ratio in Eq. 25 for $\mathcal{N} \equiv d_{\mathcal{L}}$. (Since by the definition, $\mathcal{N}$ is determined up to a constant by $\Gamma$, we can assume that $\mathcal{N}$ is as in Eq. 8). Furthermore, the direct computations, cf. [3, Chapter 5], give us that in the standard notation $p=(z, t)$ for coordinates of a point $p \in \mathbb{H}_{1}$ it holds that:

$$
\left|\nabla_{0} \mathcal{N}(z, t)\right|^{2}=\frac{|z|^{2}}{\sqrt{|z|^{4}+t^{2}}} .
$$

Next, let us assume that $u$ is super (sub) solution, i.e. $\mathcal{L} u \geq(\leq) 0$ in $\Omega$, respectively. Then, by combining Eqs. 25 and 26 together with Eq. 27 we obtain the following condition to be satisfied by $u$ :

$u(p)=\frac{1}{\left|B_{d}(p, r)\right|} \int_{B_{d}(p, r)} u(q) d q \leq(\geq) \frac{4}{\pi r^{4}} \int_{B_{d_{\mathcal{L}}}(p, r)} \frac{\left|z\left(p^{-1} q\right)\right|^{2}}{\sqrt{\left|z\left(p^{-1} q\right)\right|^{4}+\left|t\left(p^{-1} q\right)\right|^{2}}} u(q) d q$,

where $z(\cdot)$ and $t(\cdot)$ stand for, respectively, the $z$ - and $t$-coordinates of a point in $\mathbb{H}_{1}$, see Section 5. Moreover, note that

$$
\frac{\left|B_{d_{\mathcal{L}}}(p, r)\right|}{\left|B_{d}(p, r)\right|}=\frac{\pi / 4}{\left|B_{d}(0,1)\right|}:=C_{d, d_{\mathcal{L}}}<\infty
$$

due to the left-invariance of the Hausdorff measure on $\mathbb{H}_{1}$. Hence, the necessary condition for $u$ to satisfy $\mathcal{L} u \geq(\leq) 0$ in $\Omega$ is

$$
C_{d, d_{\mathcal{L}}}\|u\|_{L^{1}\left(B_{d}(p, r)\right)} \leq(\geq)\left\|u\left|\nabla_{0} \mathcal{N}\right|^{2} \circ \tau_{p^{-1}}\right\|_{L^{1}\left(B_{d_{\mathcal{L}}}(p, r)\right)} \quad \text { for all } p \in \Omega .
$$

\section{Harmonicity on $\mathbb{H}_{1}$}

The purpose of this section is to provide a large class of $\mathcal{L}$-harmonic functions which in the same time are also strongly harmonic with respect to the pseudodistance derived from an $\mathcal{L}$-gauge. Namely, a subset of the so-called spherical harmonic polynomials, called for short, spherical harmonics. It is, perhaps, surprising that such a class exists, if one takes 
into account that a spherical harmonic function must satisfy two kinds of the mean value property: the one in Definition 3.2 and Eq. 25. First, we recall the necessary definitions and set up the stage for the main computations of the mean value property for a class of spherical harmonics. Then, we present an example of a spherical harmonic function (and hence a $\mathcal{L}$ harmonic function) which fails to be strongly harmonic, see Example 6. Finally, we address an open question about identifying all spherical harmonics which are strongly harmonic.

The discussion below is, in fact, valid for all the Heisenberg groups $\mathbb{H}_{n}$, however for simplicity we restrict to the case $n=1$. Recall, that on $\mathbb{H}_{1}$ one introduces the coordinates $(z, t)$ where $z=x+i y \in \mathbb{C}, t \in \mathbb{R}$ and the multiplication is defined by

$$
\begin{aligned}
\left(z_{1}, t_{1}\right)\left(z_{2}, t_{2}\right) & =\left(z_{1}+z_{2}, t_{1}+t_{2}+2 \operatorname{Im}\left(z_{1} \bar{z}_{2}\right)\right) \\
& =\left(x_{1}+x_{2}, y_{1}+y_{2}, t_{1}+t_{2}+2\left(x_{2} y_{1}-x_{1} y_{2}\right)\right) .
\end{aligned}
$$

We observe that $(z, t)^{-1}=(-z,-t)$.

Since the Dirichlet problem for $\mathcal{L}$ is solvable on $B(0,1)$, and $\mathcal{L}$ is analytically hypoelliptic (see [13] and the references therein), there is a family of $\mathcal{L}$-harmonic polynomials which play the same role in $\mathbb{H}_{1}$ as do the spherical harmonics in $\mathbb{R}^{n}$. By analytic hypoellipticity, any harmonic function on $B(0,1)$ is real analytic and the "spherical harmonics" are naturally defined in terms of their homogeneous degree.

Definition 5.1 An $\mathcal{L}$-spherical harmonic of degree $\ell=0,1,2, \ldots$, is a polynomial in $z, \bar{z}$ and $t$, which is $\mathcal{L}$-harmonic and homogeneous of degree $\ell$ with respect to the Heisenberg dilation.

Using Korányi's formula in [16], a basis for the $\mathcal{L}$-harmonic polynomials of homogeneous degree $2 m+k+l$ can be enumerated in the form

$$
P_{k, l}^{m}(z, t)=r_{k, l}^{m}\left(t+i|z|^{2}, t-i|z|^{2}\right) z^{k} \bar{z}^{l},
$$

where the polynomial $z^{k} \bar{z}^{l}$ is $\mathcal{L}$-harmonic and

$$
r_{k, l}^{m}(w, \bar{w})=m ! \sum_{j=0}^{m} C(l, j) C(k, m-j) w^{m-j} \bar{w}^{j}
$$

with

$$
C(l, j)=\left\{\begin{array}{l}
1 \quad \text { if } \quad j=0 \\
\frac{1}{j !} \prod_{i=0}^{j-1}\left(\frac{1}{2}+l+i\right) \quad \text { if } \quad j>0 .
\end{array}\right.
$$

Hence, the $\mathcal{L}$-harmonic functions $u$ on $B(0,1)$ have the form

$$
u(z, t)=\sum_{k, l, m} a_{k, l, m} P_{k, l}^{m}(z, t) .
$$

On $\mathbb{H}_{1}$, the $\mathcal{L}$-harmonic polynomials of the form $z^{k} \bar{z}^{l}$ are precisely those for which $k=0$ or $l=0$, and so a basis for the $\mathcal{L}$-harmonic polynomials of homogeneous degree $2 m+k$ is given by elements of the form

$$
P_{0, k}^{m}(z, t)=r_{0, k}^{m}\left(t+i|z|^{2}, t-i|z|^{2}\right) \bar{z}^{k} \quad \text { or } \quad P_{k, 0}^{m}(z, t)=r_{k, 0}^{m}\left(t+i|z|^{2}, t-i|z|^{2}\right) z^{k} .
$$

We are now in a position to state the key observation, shown by direct calculation.

Observation 5.1 The following spherical harmonic polynomials on $\mathbb{H}_{1}$ are strongly harmonic for all $k \in \mathbb{N}$ :

$$
P_{0, k}^{1}(z, t)=\left((1+k) t+i k|z|^{2}\right) z^{k} \quad \text { and } \quad P_{k, 0}^{1}(z, t)=\left((1+k) t-i k|z|^{2}\right) \bar{z}^{k} .
$$


Proof The proof relies on using the cylindrical coordinates. Notice, that since $P_{k, 0}^{1}(z, t)=$ $\overline{P_{0, k}^{1}(z, t)}$, it suffices to check that $P_{k, 0}^{1}(z, t)$ is strongly harmonic for all $k$.

If $p=\left(z_{0}, t_{0}\right)$ and $q=(z, t)$, then we have

$$
P_{0, k}^{1}(p q)=\left((1+k)\left(t+t_{0}+2 \operatorname{Im}\left(z_{0} \bar{z}\right)\right)+i k\left|z+z_{0}\right|^{2}\right)\left(z+z_{0}\right)^{k} .
$$

In cylindrical coordinates $(z, t)=\left(r e^{i \theta}, t\right)$ and the above function takes the following form:

$$
\begin{aligned}
P_{0, k}^{1}\left(p\left(r e^{i \theta}, t\right)\right)= & \left((1+k)\left(t_{0}+t+2 r\left(y_{0} \cos (\theta)-x_{0} \sin (\theta)\right)\right)\left(r e^{i \theta}+z_{0}\right)^{k}\right. \\
& +i k\left(r^{2}+2 r\left(x_{0} \cos (\theta)+y_{0} \sin (\theta)\right)+x_{0}^{2}+y_{0}^{2}\right)\left(r e^{i \theta}+z_{0}\right)^{k} .
\end{aligned}
$$

Moreover, for any ball $B(p, R) \subset G$ we have:

$\int_{B(p, R)} P_{0, k}^{1}(q) d q=\int_{B(0, R)} P_{0, k}^{1}(p q) d q=\int_{0}^{R} \int_{-\sqrt{R^{4}-r^{4}}}^{\sqrt{R^{4}-r^{4}}} \int_{-\pi}^{\pi} P_{0, k}^{1}\left(p\left(r e^{i \theta}, t\right)\right) d \theta d t r d r$.

In view of Eq. 29), inner integral $\int_{-\pi}^{\pi} P_{0, k}^{1}\left(p\left(r e^{i \theta}, t\right)\right) d \theta$ requires the following integrals which we evaluate with residues:

$$
\begin{aligned}
& \int_{-\pi}^{\pi}\left(r e^{i \theta}+z_{0}\right)^{k} d \theta=\int_{S(0, r)}\left(z+z_{0}\right)^{k} \frac{d z}{i z}=2 \pi z_{0}^{k}, \\
& \int_{-\pi}^{\pi} \cos (\theta)\left(r e^{i \theta}+z_{0}\right)^{k} d \theta=r^{k} \\
& \int_{-\pi}^{\pi} \cos (\theta)\left(e^{i \theta}+\frac{z_{0}}{r}\right)^{k} d \theta=r^{k} \int_{S(0,1)} \frac{1}{2}\left(z+z^{-1}\right)\left(z+r^{-1} z_{0}\right)^{k} \frac{d z}{i z}=k \pi r z_{0}^{k-1}, \\
& \int_{-\pi}^{\pi} \sin (\theta)\left(r e^{i \theta}+z_{0}\right)^{k} d \theta=r^{k} \int_{S(0,1)} \frac{1}{2 i}\left(z-z^{-1}\right)\left(z+r^{-1} z_{0}\right)^{k} \frac{d z}{i z}=i k \pi r z_{0}^{k-1} .
\end{aligned}
$$

It now follows from Eq. 29 that

$$
\int_{-\pi}^{\pi} P_{0, k}^{1}\left(p\left(r e^{i \theta}, t\right)\right) d \theta=2 \pi\left((1+k)\left(t_{0}+t\right)+i k\left|z_{0}\right|^{2}\right) z_{0}^{k} .
$$

The remaining integrations are straight forward, as we have

$$
\int_{-\sqrt{R^{4}-r^{4}}}^{\sqrt{R^{4}-r^{4}}} \int_{-\pi}^{\pi} P_{0, k}^{1}\left(p\left(r e^{i \theta}, t\right)\right) d \theta d t=4 \pi\left((k+1) t_{0}+i k\left|z_{0}\right|^{2}\right) z_{0}^{k} \sqrt{R^{4}-r^{4}}
$$

and

$$
\begin{aligned}
\int_{0}^{R} \int_{-\sqrt{R^{4}-r^{4}}}^{\sqrt{R^{4}-r^{4}}} \int_{-\pi}^{\pi} P_{0, k}^{1}\left(p\left(r e^{i \theta}, t\right)\right) d \theta d t r d r & =4 \pi\left((k+1) t_{0}+i k\left|z_{0}\right|^{2}\right) z_{0}^{k} \int_{0}^{R} \sqrt{R^{4}-r^{4}} r d r \\
& =4 \pi\left((k+1) t_{0}+i k\left|z_{0}\right|^{2}\right) z_{0}^{k} \frac{\pi}{8} R^{4} .
\end{aligned}
$$

Since

$$
\int_{B(p, R)} d q=\frac{\pi^{2}}{2} R^{4}
$$

we obtain that

$$
P_{0, k}^{1}\left(\left(z_{0}, t_{0}\right)\right)=f_{B(p, R)} P_{0, k}^{1}(q) d q
$$

and hence, $P_{0, k}^{1}$ is strongly harmonic in $\mathbb{H}_{1}$. 
Example 6 The first spherical harmonic that is not strongly harmonic is

$$
P_{0,0}^{2}(z, t)=2 t^{2}-|z|^{4} .
$$

Indeed, in this case computations similar to the one in the proof of the observation reveal that

$$
f_{B(p, R)} P_{0,0}^{2}(q) d q=P_{0,0}^{2}(p)+\frac{R^{4}}{4} .
$$

Computation with MAPLE up to homogenous degree 40 revealed no strongly harmonic spherical harmonics with the order of $t$ greater than 1 . Thus, one might suspect that strongly harmonic spherical harmonics are precisely those with the order of $t$ is less or equal to 1 and a computational proof similar to the above might reveal it to be true. However, the computations become cumbersome due to the combinatorics arising from the coefficients $C(l, j)$ at Eq. 28 and repeated use of the binomial formula. Considering the mean values at 0 does not simplify the task, indeed all the spherical harmonics are strongly harmonic at 0 .

For example let us consider

$$
P_{k, 0}^{m}(z, t)=r_{k, 0}^{m}\left(t+i r^{2}, t-i r^{2}\right) z^{k} .
$$

As above, let us apply the cylindrical coordinates $\left(r e^{i \theta}, t\right)$ and denote $z:=r w$ for $w \in$ $S(0,1)$. Upon considering the mean value of $P_{k, 0}^{m}$ at $p=0$, it follows that

$$
\begin{aligned}
& \int_{-\pi}^{\pi} P_{k, 0}^{m}\left(r e^{i \theta}, t\right) d \theta=\int_{S(0,1)} P_{k, 0}^{m}(r w, t) \frac{d w}{i w}=-i r^{k} r_{k, 0}^{m}\left(t+i r^{2}, t-i r^{2}\right) \\
& \int_{S(0,1)} w^{k-1} d w=0, \quad \text { for } k \geq 1 .
\end{aligned}
$$

Therefore, the mean value property for $P_{k, 0}^{m}$ holds at the origin.

It is interesting to note that if the strongly harmonic spherical harmonics are exactly the spherical harmonics of $t$ degree less or equal to one, then strongly harmonic spherical harmonics are solutions to the Beltrami-Laplace equation

$$
\tilde{X}^{2} u+\tilde{Y}^{2} u+\frac{\partial^{2} u}{\partial t^{2}}=0 .
$$

Moreover, by analytic hypoellipticity, the same can be said for strongly harmonic functions on $B(0,1)$.

In view of results of this section we pose the following problems.

\section{Open Problems}

(1) Identify all spherical harmonic polynomials $P_{k, 0}^{m}$ that are strongly harmonic.

(2) Describe other classes of functions in Carnot-Carathéodory groups that are both $\mathcal{L}$ harmonic and strongly harmonic.

\section{Determining Set}

Let $\Omega \subset \mathbb{H}_{1}$. We say that $S \subset \Omega$ is the determining set if for any $u: \Omega \rightarrow \mathbb{R}$ strongly harmonic in the set $S$, it follows that $u \in \mathcal{H}(\Omega)$. For the studies of determining sets for harmonic functions in the Euclidean setting we refer to Flatto [8]. In this section we show that for continuous functions it is enough to assume the mean value property on a dense 
subset of $\Omega$ in order to infer the harmonicity in the whole domain $\Omega$. The additional technical assumption is that $\mathbb{H}_{1}$ must be a geodesic space with respect to the underlying metric $d$.

Observation 6.1 Let $S \subset \Omega$ be dense in $\Omega$. Furthermore, let us suppose that metric $d$ on $\mathbb{H}_{1}$ is such that $\mathbb{H}_{1}$ is geodesic space with respect to $d$. Then it holds that, if $u \in C(\Omega)$ and satisfies the mean-value property (9) at points of $S$, then $u \in \mathcal{H}(\Omega)$.

Proof Let $p \in \Omega \backslash S$ and $\left(p_{n}\right)$ be a sequence of points in $S$ such that $p_{n} \rightarrow p$, as $n \rightarrow \infty$ with respect to the given metric $d$. Let $\epsilon>0$ and $N$ be such that for all $n>N$ it holds that

$$
-\epsilon+u\left(p_{n}\right) \leq u(p) \leq \epsilon+u\left(p_{n}\right),
$$

by continuity of $u$ at $p$. Then, the following estimate holds for any $r>0$ and all balls $B\left(p_{n}, r\right) \Subset \Omega, B(p, r) \Subset \Omega:$

$$
\begin{aligned}
u(p) \leq \epsilon+u\left(p_{n}\right) & =\epsilon+\frac{1}{\left|B_{d}(p, r)\right|} \frac{\left|B_{d}(p, r)\right|}{\left|B_{d}\left(p_{n}, r\right)\right|}\left(\int_{B_{d}(p, r)} u(q)+\int_{B_{d}(p, r) \backslash B_{d}\left(p_{n}, r\right)} u(q)\right) \\
& \leq \epsilon+\frac{\left|B_{d}(p, r)\right|}{\left|B_{d}\left(p_{n}, r\right)\right|} u_{B_{d}(p, r)}+\int_{B_{d}(p, r) \Delta B_{d}\left(p_{n}, r\right)} u(q),
\end{aligned}
$$

where $B_{d}(p, r) \Delta B_{d}\left(p_{n}, r\right)$ stands for the symmetric difference between balls. Observe that $\frac{\left|B_{d}(p, r)\right|}{\left|B_{d}\left(p_{n}, r\right)\right|} \rightarrow 1$, as $n \rightarrow \infty$. Moreover, since $\mathbb{H}_{1}$, equipped with metric $d$, is a geodesic space, then by Proposition 4.1, the Lebesgue measure is metrically continuous with respect to $d$, cf. Eq. 10 above, see also Definition 2.1 and the discussion in [1, Section 2] for further details. In particular, the following symmetric difference satisfies condition (10):

$$
\left|B_{d}(p, r) \Delta B_{d}\left(p_{n}, r\right)\right| \rightarrow 0, \quad \text { as } n \rightarrow \infty .
$$

By applying the Lebesgue convergence theorem we conclude that the second integral in Eq. 30 converges to 0 , while the first one to mean value of $u$ at $p$. Note that, since $u \in$ $L_{l o c}^{1}(\Omega)$, then by invoking again the metric continuity of the measure with respect to $d$, one obtains that

$$
\left|\int_{B_{d}(p, r) \Delta B_{d}\left(p_{n}, r\right)} u(q)\right| \rightarrow 0, \quad \text { as } n \rightarrow \infty .
$$

Thus, we also get that $u(p) \geq-\epsilon+u_{B_{d}(p, r)}$ and the arbitrary choice of $\epsilon$ allows us to conclude that $u(p)=u_{B_{d}(p, r)}$. Hence, the proof is completed.

Acknowledgments We would like to thank the anonymous referees for their comments and suggestions which improved the quality of the presentation.

Open Access This article is distributed under the terms of the Creative Commons Attribution 4.0 International License (http://creativecommons.org/licenses/by/4.0/), which permits unrestricted use, distribution, and reproduction in any medium, provided you give appropriate credit to the original author(s) and the source, provide a link to the Creative Commons license, and indicate if changes were made.

\section{Appendix: The Proof of Theorem 4.4}

In the Appendix we prove Theorem 4.4, which states that $\mathcal{L}$-harmonic functions satisfy a variant of the mean value property with respect to kernels defined via gradient of pseudonorm given by the fundamental solution of $\mathcal{L}$. We begin with preliminaries regarding the representation of $\mathcal{L}$ in the divergence form. 
Fix an orthonormal basis $\left\{E_{i}\right\}_{i=1}^{N}$ such that $\mathfrak{g}^{1}=\operatorname{span}\left\{E_{i} \mid i=1, \ldots, N_{1}\right\}$ and

$$
\tilde{X}_{i} u(p)=\left.\frac{d}{d t} u\left(p \exp \left(t E_{i}\right)\right)\right|_{t=0} \quad \text { for } i=1,2, \ldots, N .
$$

Recall that $N=N_{1}+\ldots+N_{s}$, cf. Definition 2.1. If $x_{i}$, for $i=1,2, \ldots, N$ denote the coordinates on $G$ induced by the chosen basis of $\mathfrak{g}$ via the exponential map, then

$$
\tilde{X}_{i} u=\sum_{j=1}^{N} d x_{j}\left(\tilde{X}_{i}\right) \frac{\partial}{\partial x_{j}} .
$$

In coordinates we have

$$
\begin{aligned}
\mathcal{L} u=\sum_{i=i_{0}}^{N_{1}} \tilde{X}_{i}^{2} u & =\sum_{j=1}^{N} \sum_{k=1}^{N} \sum_{i=1}^{N_{1}} d x_{j}\left(\tilde{X}_{i}\right) \frac{\partial}{\partial x_{j}}\left(d x_{k}\left(\tilde{X}_{i}\right) \frac{\partial u}{\partial x_{k}}\right) \\
& =\sum_{j=1}^{N} \sum_{k=1}^{N} \sum_{i=1}^{N_{1}} \frac{\partial}{\partial x_{j}}\left(d x_{j}\left(\tilde{X}_{i}\right) d x_{k}\left(\tilde{X}_{i}\right) \frac{\partial u}{\partial x_{k}}\right) \\
& =\operatorname{div} A \nabla u,
\end{aligned}
$$

where the coefficients of matrix $A$ are given by the formula $A_{j, k}=\sum_{i=1}^{N_{1}} d x_{j}\left(\tilde{X}_{i}\right) d x_{k}\left(\tilde{X}_{i}\right)$ for $j, k=1, \ldots, N$. Note that the third equality (31) uses the identity $\frac{\partial}{\partial x_{j}}\left(d x_{j}\left(\tilde{X}_{i}\right)\right)=0$ for each $i=1, \ldots, N_{1}$ which follows from the nilpotency of $G$.

Expanding in coordinates as above we also get $\left\langle\nabla_{0} u, \nabla_{0} u\right\rangle_{G}=\langle A \nabla u \nabla u\rangle_{\mathbb{R}^{N}}$ and note that a mapping $(p, q) \rightarrow\left\langle\nabla_{0} u, \nabla_{0} u\right\rangle\left(p^{-1} q\right)$ is left invariant and homogeneous of degree 0 with respect to dilation since $\nabla_{0} u$ has degree 0 .

Proof of Theorem 4.4 By direct calculation, it is easy to check that the following Green's identity holds:

$$
v \mathcal{L} u-u \mathcal{L} v=\operatorname{div}(v A \nabla u-u A \nabla v) .
$$

Since $u$ is $\mathcal{L}$-harmonic in $\Omega \subset G$ and $v=\mathcal{N}^{2-Q} \circ \tau_{p^{-1}}=-\Gamma \circ \tau_{p^{-1}}$, then $v$ is $\mathcal{L}$-harmonic on $G \backslash\{p\}$ and

$$
\int_{B(p, r) \backslash B(p, \epsilon)} \operatorname{div}(v A \nabla u-u A \nabla v)(q) d q=0 .
$$

Applying Stokes theorem gives

$$
\int_{\partial B(p, r)} u A \nabla v(v) \cdot n(p, v) d S(v)=\int_{\partial B(p, \epsilon)} u A \nabla v(v) \cdot n(p, v) d S(v),
$$

where $d S$ is defined via the Gramm determinant. Note that we have used the following consequences of the choice of $u$ and $v$ :

$$
\begin{aligned}
\int_{\partial B(p, \rho)} v A \nabla u(v) \cdot n(p, v) d S(v) & =\rho^{2-Q} \int_{\partial B(p, \rho)} A \nabla u(v) \cdot n(p, v) d S(v) \\
& =\rho^{2-Q} \int_{\partial B(p, \rho)} \operatorname{div} A \nabla u(q) d q \\
& =\rho^{2-Q} \int_{\partial B(p, \rho)} \mathcal{L} u(q) d q=0 .
\end{aligned}
$$


We define the following kernel $K(p, q)$, cf. Definition 5.5.1 and the proof of Theorem 5.5.4 in [3]:

$K(p, q):=(2-Q) \mathcal{N}\left(p^{-1} q\right)^{1-Q} \frac{\left\langle\nabla_{0} \mathcal{N}\left(p^{-1} q\right), \nabla_{0} \mathcal{N}\left(p^{-1} q\right)\right\rangle}{\left\|\nabla\left(\mathcal{N} \circ \tau_{p^{-1}}\right)(q)\right\|}:=(2-Q) \mathcal{N}\left(p^{-1} q\right)^{1-Q} \mathcal{K}(p, q)$,

where $p, q \in G$ and $p \neq q$. Next, we define

$$
T_{r}(u)(p):=\int_{\partial B(p, r)} u(q) K(p, q) d S(q) \text { for any } p \in G,
$$

where $r>0$ is such that $B(p, r) \Subset \Omega$. It turns out that transform $T_{r}$ satisfies the mean value property. Namely, by Eq. 32 we have $T_{r}(u)(p)=T_{\epsilon}(u)(p)$ for all $0<\epsilon<r$. It then follows that

$$
\begin{aligned}
\left|T_{r}(u)(p)-u(p) T_{r}\left(\chi_{\Omega}\right)(p)\right| & \leq \int_{\partial B(p, \epsilon)}|u(q)-u(p) \| K(p, q)| d S(q) \\
& \leq \frac{1}{Q-2} \sup _{\partial B(p, \epsilon)}\left|u(q)-u(p) \| T_{r}\left(\chi_{\Omega}\right)(p)\right| .
\end{aligned}
$$

The continuity of $u$ implies the following:

$$
T_{r}(u)(p)=u(p) T_{r}\left(\chi_{\Omega}\right)(p) .
$$

Furthermore, since $T_{r}(u)(p)=T_{t}(u)(p)$ for all $t<r$, we have

$$
\begin{aligned}
T_{r}(u)(p) r^{Q} & =T_{r}(u)(p) \int_{0}^{r} Q t^{Q-1} d t=\int_{0}^{r} T_{t}(u)(p) Q t^{Q-1} d t \\
& =\int_{0}^{r} \int_{\partial B(p, t)} u(v) K(p, v) d S(v) Q t^{Q-1} d t \\
& =Q(2-Q) \int_{0}^{r} \int_{\partial B(p, t)} u(v) \mathcal{K}(p, v) d S(v) d t \\
& =Q(2-Q) \int_{B(p, r)} u(q) \mathcal{K}(p, q)\left\|\nabla\left(\mathcal{N} \circ \tau_{p^{-1}}\right)(q)\right\| d q \\
& =Q(2-Q) \int_{B(p, r)} u(q)\left\langle\nabla_{0} \mathcal{N}\left(p^{-1} q\right), \nabla_{0} \mathcal{N}\left(p^{-1} q\right)\right\rangle d q .
\end{aligned}
$$

Finally, from Eq. 33 we get the assertion of Theorem 4.4 for all $p \in G$ and all balls $B(p, r) \Subset \Omega$ :

$$
u(p)=\frac{\int_{B(p, r)} u(q)\left|\nabla_{0} \mathcal{N}\left(p^{-1} q\right)\right|^{2} d q}{\int_{B(p, r)}\left|\nabla_{0} \mathcal{N}\left(p^{-1} q\right)\right|^{2} d q}=\frac{\int_{B(0, r)} u(p q)\left|\nabla_{0} \mathcal{N}(q)\right|^{2} d q}{\int_{B(0, r)}\left|\nabla_{0} \mathcal{N}(q)\right|^{2} d q} .
$$

As mentioned in Section 4, these computations indicate that $\mathcal{L}$-harmonic functions need not necessarily be strongly harmonic.

\section{References}

1. Adamowicz, T., Gaczkowski, M., Górka, P.: Harmonic functions on metric measure spaces. Rev. Mat. Complut. (2018). https://doi.org/10.1007/s13163-018-0272-7 
2. Adamowicz, T., Warhurst, B.: Three-spheres theorems for subelliptic quasilinear equations in Carnot groups of Heisenberg-type. Proc. Amer. Math. Soc. 144(10), 4291-4302 (2016)

3. Bonfiglioli, A., Lanconelli, E., Uguzzoni, F.: Stratified Lie Groups and Potential Theory for Their Sublaplacians. Springer Monographs in Mathematics, Springer (2007)

4. Capogna, L.: Regularity of quasi-linear equations in the Heisenberg group. Comm. Pure Appl. Math. 50(9), 867-889 (1997)

5. Capogna, L.: Regularity for quasilinear equations and 1-quasiconformal maps in Carnot groups. Math. Ann. 313(2), 263-295 (1999)

6. Capogna, L., Cowling, M.: Conformality and Q-harmonicity in Carnot groups. Duke Math. J. 135(3), 455-479 (2006)

7. Chen, Z.-Q.: On notions of harmonicity. Proc. Amer. Math. Soc. 137(10), 3497-3510 (2009)

8. Flatto, L.: The Converse of Gauss's Theorem for Harmonic Functions. J. Diff. Eq. 1, 483-490 (1965)

9. Folland, G.B.: Subelliptic estimates and function spaces on nilpotent Lie groups. Ark. Mat. 13(2), 161207 (1975)

10. Folland, G.B., Stein, E.M.: Hardy Spaces on Homogeneous Groups. Princeton University, Mathematical Notes (1982)

11. Gaczkowski, M., Górka, P.: Harmonic functions on metric measure spaces: Convergence and compactness. Potential Anal. 31, 203-214 (2009)

12. Gilbarg, D., Trudinger, N.S.: Elliptic Partial Differential Equations of Second Order. Springer-Verlag (1983)

13. Greiner, P.C.: Spherical harmonics on the Heisenberg group. Canad. Math. Bull. 23(4), 383-396 (1980)

14. Hörmander, L.: Hypoelliptic second order differential equations. Acta Math. 119, 147-71 (1967)

15. Kellogg, O.: Foundations of potential theory, Reprint from the first edition of 1929. Die Grundlehren der Mathematischen Wissenschaften Band, vol. 31. Springer-Verlag, Berlin-New York (1967)

16. Korányi, A.: Kelvin transforms and harmonic polynomials on the Heisenberg group. J. Funct. Anal. 45(2), 293-296 (1982)

17. Manfredi, J., Mingione, G.: Regularity results for quasilinear elliptic equations in the Heisenberg group. Math. Ann. 339(3), 485-544 (2007)

18. Ricciotti, D.: p-Laplace Equation in the Heisenberg Group. Regularity of solutions, SpringerBriefs in Mathematics. BCAM SpringerBriefs. Springer, [Cham]; BCAM Basque Center for Applied Mathematics, Bilbao, (2015), pp. xiv+87

19. Tessera, R.: Large scale Sobolev inequalities on metric measure spaces and applications. Rev. Mat. Iberoam. 24(3), 825-864 (2008) 